STRUCTURAL BIOLOGY

ISSN 2059-7983

Received 4 September 2019

Accepted 4 December 2019

Keywords: refinement of protein structures; quantum-mechanical calculations; neutrons; corrigendum.

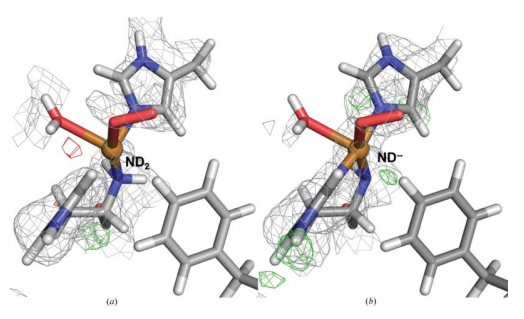

OPEN $\odot$ ACCESS

\section{Refinement of protein structures using a combination of quantum-mechanical calculations with neutron and X-ray crystallographic data. Corrigendum}

\author{
Octav Caldararu, ${ }^{a}$ Francesco Manzoni, ${ }^{a, b}$ Esko Oksanen, ${ }^{b, c}$ Derek T. Logan ${ }^{b}$ and \\ Ulf Ryde ${ }^{\mathrm{a} *}$
}

aDivision of Theoretical Chemistry, Lund University, Chemical Centre, PO Box 124, SE-221 00, Lund, Sweden, ${ }^{\mathbf{b}}$ Department of Biochemistry and Structural Biology, Lund University, Chemical Centre, PO Box 124, SE-221 00, Lund, Sweden, and ${ }^{c}$ European Spallation Source ESS ERIC, PO Box 176, SE-221 00, Lund, Sweden. *Correspondence e-mail: ulf.ryde@teokem.lu.se

Corrections are published for the article by Caldararu et al. [(2019), Acta Cryst. D75, 368-380].

In our previous article, Refinement of protein structures using a combination of quantum-mechanical calculations with neutron and X-ray crystallographic data (Caldararu, Manzoni et al., 2019), there was a small technical error in calculating the nuclear scattering-length density maps for Fig. 11 and Table 4.

Joint X-ray-neutron quantum refinement of lytic polysaccharide monooxygenase (LPMO) was conducted even though the unit-cell parameters of the X-ray crystal and of the neutron crystal are not exactly equal. The quantum refinement calculations were performed in the X-ray unit cell, but the nuclear density maps in Fig. 11 were calculated in the neutron unit cell. We report below the corrected figure (Fig. 1), with nuclear density maps calculated in the X-ray unit cell. The RSZD (real-space difference-density $Z$ ) values used in weight determination for the LPMO quantum refinement in the original article were also calculated from the maps in the wrong unit cell. A corrected table with the maximum absolute RSZD of the residues of the active site is shown below (Table 1). It can be seen that the absolute values of the RSZD scores have changed somewhat (by up to 1.2), but the variation with the X-ray and neutron weight factors, $w_{\mathrm{N}}$ and $w_{\mathrm{X}}$, does not change much. All RSZD scores are almost constant for $w_{\mathrm{N}}=$ $w_{\mathrm{X}}=1-10$, whereas RSZD increases if the weights are further decreased. Therefore, the selection of $w_{\mathrm{N}}=w_{\mathrm{X}}=1$ as proper weights is still in accordance with the RSZD values estimated from maps calculated in the correct unit cell.

In the corrected version of the figure, there is no positive difference density at the deprotonated $\mathrm{N}$-terminus at the $3.0 \sigma$ level. However, there is still positive difference density around the N-terminus at the $2.8 \sigma$ level as can be seen in the corrected Fig. 1(b). This level is still well above the noise level of the nuclear density maps in that area $(\sim 2.3 \sigma)$. Although the maps without the D2 atom at the $\mathrm{N}$-terminus look more similar to the ones reported by Bacik et al. (Bacik et al., 2017), the structures with two deuterium atoms still fit better to the neutron data. Thus, our conclusions about the use of quantum 


\section{addenda and errata}

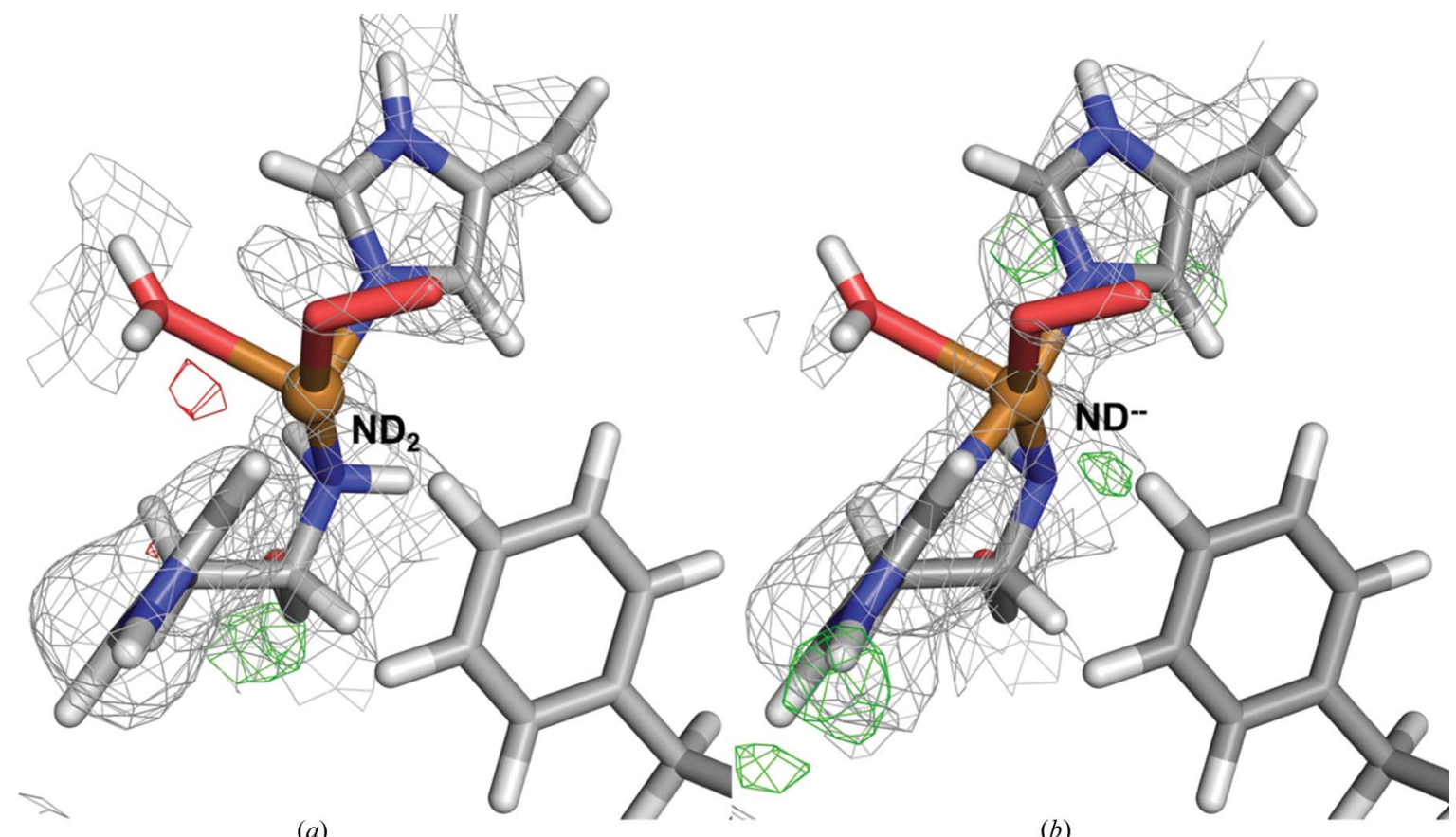

(a)

(b)

Figure 1

Structure and nuclear density maps of the active site of LPMO in subunit $B$ after QM refinement. $(a)$ The N-terminus in the protonated $-\mathrm{ND}_{2}$ form and (b) the N-terminus in the deprotonated $\mathrm{ND}^{-}$form. The $2 m\left|F_{\mathrm{o}}\right|-D\left|F_{\mathrm{c}}\right|$ nuclear density at $1.0 \sigma$ is shown as a grey grid and $m\left|F_{\mathrm{o}}\right|-D\left|F_{\mathrm{c}}\right|$ nuclear difference density is shown at $2.8 \sigma$ (green grid) and $-2.8 \sigma$ (red grid). This is a corrected version of Fig. 11 from Caldararu, Manzoni et al. (2019).

Table 1

Maximum absolute RSZD values of the residues in the QM system in the QM refinement of subunit $A$ of the LPMO structure obtained with different weights of the experimental data $\left(w_{\mathrm{N}}\right.$ and $\left.w_{\mathrm{X}}\right)$.

This is a corrected version of Table 4 from Caldararu, Manzoni et al. (2019). Note that $w_{\mathrm{X}} / w_{\mathrm{N}}=1$ in all refinements.

\begin{tabular}{llllllllllllll}
\hline$w_{\mathrm{N}}$ & 0.001 & 0.01 & 0.1 & 1 & 2 & 3 & 4 & 5 & 6 & 7 & 8 & 9 \\
\hline$w_{\mathrm{X}}$ & 0.001 & 0.01 & 0.1 & 1 & 2 & 3 & 4 & 5 & 6 & 7 & 8 & 9 \\
\hline Cu & 0.4 & 0.4 & 0.4 & 0.4 & 0.4 & 0.4 & 0.4 & 0.4 & 0.4 & 0.4 & 0.4 & 0.4 & 0.4 \\
Peroxide & 0.3 & 0.3 & 0.1 & 0.1 & 0.1 & 0.0 & 0.0 & 0.0 & 0.0 & 0.0 & 0.0 & 0.0 & 0.0 \\
His32 & 1.7 & 1.5 & 0.9 & 0.4 & 0.4 & 0.4 & 0.4 & 0.4 & 0.4 & 0.4 & 0.4 & 0.4 & 0.4 \\
His109 & 1.0 & 1.0 & 0.8 & 0.7 & 0.7 & 0.7 & 0.7 & 0.7 & 0.7 & 0.7 & 0.7 & 0.7 & 0.7 \\
Phe164 & 1.4 & 1.4 & 1.4 & 1.4 & 1.4 & 1.4 & 1.4 & 1.4 & 1.4 & 1.4 & 1.4 & 1.4 & 1.4 \\
Wat301 & 1.7 & 1.7 & 1.8 & 1.7 & 1.7 & 1.7 & 1.7 & 1.7 & 1.7 & 1.7 & 1.7 & 1.7 & 1.7 \\
Sum & $\mathbf{6 . 5}$ & $\mathbf{6 . 3}$ & $\mathbf{5 . 5}$ & $\mathbf{4 . 7}$ & $\mathbf{4 . 7}$ & $\mathbf{4 . 6}$ & $\mathbf{4 . 6}$ & $\mathbf{4 . 6}$ & $\mathbf{4 . 6}$ & $\mathbf{4 . 6}$ & $\mathbf{4 . 6}$ & $\mathbf{4 . 6}$ & $\mathbf{4 . 6}$ \\
\hline
\end{tabular}

refinement in elucidating protonation states in metalloenzymes do not change.

Most importantly, the correction in no way affects the combined quantum mechanical and joint X-ray-neutron refinement method, which was presented in the article. The LPMO quantum refinement was included only as a second application of the method, taken from another publication (Caldararu, Oksanen et al., 2019a), for which a more detailed correction has already been submitted (Caldararu, Oksanen et al., 2019b).

\section{References}

Bacik, J.-P., Mekasha, S., Forsberg, Z., Kovalevsky, A. Y., VaajeKolstad, G., Eijsink, V. G. H., Nix, J. C., Coates, L., Cuneo, M. J., Unkefer, J. \& Chen, J. C. (2017). Biochemistry, 56, 25292532.

Caldararu, O., Manzoni, F., Oksanen, E., Logan, D. T. \& Ryde, U. (2019). Acta Cryst. D75, 368-380.

Caldararu, O., Oksanen, E., Ryde, U. \& Hedegård, E. D. (2019a). Chem. Sci. 10, 576-586.

Caldararu, O., Oksanen, E., Ryde, U. \& Hedegård, E. D. (2019b). Chem. Sci. 10, 8262-8263. 
STRUCTURAL BIOLOGY

ISSN 2059-7983

\section{Refinement of protein structures using a combination of quantum-mechanical calculations with neutron and X-ray crystallographic data}

\author{
Octav Caldararu, ${ }^{\mathrm{a}}$ Francesco Manzoni, ${ }^{\mathrm{a}, \mathrm{b}}$ Esko Oksanen, ${ }^{\mathrm{b}, \mathrm{c}}$ Derek T. Logan ${ }^{\mathrm{b}}$ and \\ Ulf Ryde ${ }^{\mathrm{a} *}$
}

Received 17 October 2018

Accepted 30 January 2019

Edited by F. Meilleur, Oak Ridge National Laboratory, USA, and North Carolina State University, USA

This article is dedicated to the memory of Francesco Manzoni, who died from a heart attack on 12 March 2017 at the age of 27.

Keywords: refinement; neutron crystallography; hydrogen atoms; quantum chemistry; galectin-3; lytic polysaccharide monooxygenase; quantum refinement.

Supporting information: this article has supporting information at journals.iucr.org/d

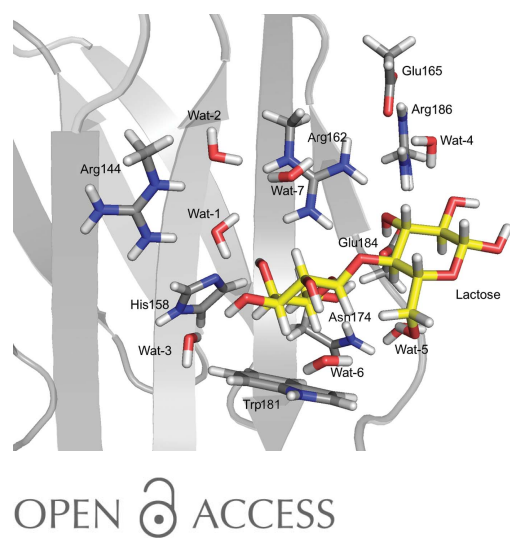

aDepartment of Theoretical Chemistry, Lund University, Chemical Centre, PO Box 124, SE-221 00 Lund, Sweden, ${ }^{\mathbf{b}}$ Department of Biochemistry and Structural Biology, Centre for Molecular Protein Science, Lund University, Chemical Centre, PO Box 124, SE-221 00 Lund, Sweden, and ' Instruments Division, European Spallation Source ESS ERIC, PO Box 176, SE-221 00 Lund, Sweden. *Correspondence e-mail: ulf.ryde@teokem.lu.se

Neutron crystallography is a powerful method to determine the positions of $\mathrm{H}$ atoms in macromolecular structures. However, it is sometimes hard to judge what would constitute a chemically reasonable model, and the geometry of $\mathrm{H}$ atoms depends more on the surroundings (for example the formation of hydrogen bonds) than heavy atoms, so that the empirical geometry information for the $\mathrm{H}$ atoms used to supplement the experimental data is often less accurate. These problems may be reduced by using quantum-mechanical calculations. A method has therefore been developed to combine quantum-mechanical calculations with joint crystallographic refinement against X-ray and neutron data. A first validation of this method is provided by re-refining the structure of the galectin-3 carbohydrate-recognition domain in complex with lactose. The geometry is improved, in particular for water molecules, for which the method leads to better-resolved hydrogen-bonding interactions. The method has also been applied to the active copper site of lytic polysaccharide monooxygenase and shows that the protonation state of the amino-terminal histidine residue can be determined.

\section{Introduction}

The majority of structural information about biological macromolecules is obtained from X-ray crystallography. An important limitation of $\mathrm{X}$-ray crystallography is that $\mathrm{H}$ atoms can only rarely be visualized in the electron-density maps, as the scattering by just one electron is too weak. Neutron crystallography (Blakeley, 2009) is a method that is able to complement the information from X-ray crystallography because neutrons are scattered by the nuclei. Moreover, deuterium, the heavier isotope of hydrogen, scatters neutrons comparably to carbon or nitrogen (Sears, 1986). Consequently, neutron structures can help to determine the positions of $\mathrm{H}$ atoms, describe the geometry of hydrogen bonds, determine the protonation and tautomeric states of ligands and pinpoint the function of water molecules. However, as the inclusion of $\mathrm{H}$ atoms in the model increases the number of parameters to be refined and neutron structures are typically at a rather low resolution, neutron data alone are typically not sufficient to define all of the atomic positions. Therefore, refinement protocols have been introduced that combine X-ray and neutron data into a single target function, against which the coordinates are refined (Coppens, 1967; Orpen et al., 1978; Wlodawer, 1980; Wlodawer \& Hendrickson, 1982; Adams et al., 2009; Afonine et al., 2010; Wlodawer \& Sjölin, 1982; Fisher et al., 2007). 
At the resolutions typical for macromolecular structures, the quality and information content of the refined structural model critically depends on prior chemical knowledge about the system, such as the amino-acid sequence and a set of chemical restraints (Cruickshank, 1999). In practice, the latter information, in the form of a molecular-mechanics (MM) or statistical force field, is used to restrain the bond lengths, angles and dihedrals in refinement, as well as to ensure proper chirality and to avoid sterical clashes. For the non-H atoms in amino acids and other standard constituents of proteins, these values are well known from a large number of high-resolution crystal structures and are available in the refinement software (Engh \& Huber, 1991). However, this is not the case for many non-protein groups, such as metal sites, substrates and inhibitors (Ryde et al., 2002; Nilsson et al., 2003; Zheng, Reimers et al., 2017; Borbulevych et al., 2016). Moreover, the geometrical information available for bonds involving $\mathrm{H}$ atoms is less reliable than for heavier atoms, even for protein components, not to mention non-protein compounds.

The restraints used in crystallographic refinement provide quite a crude description of the molecular system, as they are based on an average over many structures. The chemical environment may influence the bond lengths and angles, for example by electrostatic and induction effects, in ways that are not captured by typical restraints. The ideal geometry of hydrogen-containing bonds is particularly sensitive to the local chemical environment, which makes an averaging approach less attractive with hydrogen.

To address these issues in X-ray crystallography, we have previously developed a method to locally replace the MM restraints in the refinement by more accurate quantummechanical (QM) calculations: the quantum-refinement approach (Ryde et al., 2002; Ryde \& Nilsson, 2003; Ryde, 2007; Genoni et al., 2018). It is in principle a standard crystallographic refinement in which the MM restraints are replaced by more accurate QM calculations for a small part of the protein of particular interest, such as an active site. From a computational chemistry viewpoint, it can be seen as a combined $\mathrm{QM} / \mathrm{MM}$ calculation restrained by the crystallographic raw data. We showed that crystal structures can be locally improved using such a method, the protonation states of metal-bound water molecules can be determined and the photoreduction of metal sites can be identified (Nilsson \& Ryde, 2004; Söderhjelm \& Ryde, 2006; Rulíšek \& Ryde, 2006; Ryde \& Nilsson, 2003). The approach has been implemented in several software programs by other groups (Yu et al., 2005; Hsiao et al., 2010). In particular, Merz and Westerhoff have developed methods to combine X-ray crystallographic refinement with linear-scaling semi-empirical calculations of the whole protein and have shown that protonation states and ligand-binding conformations can be determined (Borbulevych et al., 2014, 2016; Reynolds, 2014; Yu et al., 2005). The $Q \mid R$ project also aims at refining macromolecular structures using QM methods (Zheng, Moriarty et al., 2017; Zheng, Reimers et al., 2017).

In this paper, we extend the quantum-refinement approach to neutron data, as well as combined X-ray and neutron refinement. The method is implemented in the freely available $n C N S$ software (Adams et al., 2009). As test cases, we apply the method to the refinement of the ligand-binding site of the carbohydrate-recognition domain of galectin-3 with a bound lactose molecule (Manzoni et al., 2018) and to the active copper site of lytic polysaccharide monooxygenase (LPMO).

Galectin-3 is a mammalian $\beta$-galactoside-binding protein involved in glycoprotein trafficking, signalling, cell adhesion, angiogenesis, macrophage activation and apoptosis (Leffler et al., 2002; MacKinnon et al., 2008; Delacour et al., 2009; Liu \& Rabinovich, 2010; Grigorian \& Demetriou, 2010; Johannes et al., 2018). It has been implicated in inflammation, immunity, cancer development and metastasis (Rabinovich et al., 2007).

LPMO is a metalloenzyme that is capable of inserting a single $\mathrm{O}$ atom into the glycosidic $\mathrm{C}-\mathrm{H}$ bond in polysaccharides (Vaaje-Kolstad et al., 2010). This boosts polysaccharide decomposition, which could lead to a more energy-efficient production of biofuel from abundant polysaccharides such as cellulose (Harris et al., 2014). LPMOs contain a copper ion coordinated by two histidine residues. One of the histidine residues coordinates bidentately to both the N-terminus and the imidazole side chain (Quinlan et al., 2011).

We show that the combined QM/neutron/X-ray refinement works properly and gives rise to improved structures and to an easier interpretation of ambiguous data.

\section{Methods}

\subsection{Galectin-3}

Neutron data were collected to a resolution of $1.7 \AA$ from a $1.8 \mathrm{~mm}^{3}$ crystal of perdeuterated carbohydrate-recognition domain of galectin-3 (galectin-3C) complexed with nondeuterated lactose using the LADI-III instrument at Institut LaueLangevin, Grenoble, France (Blakeley et al., 2010). X-ray data were collected to $1.0 \AA$ resolution from the same crystal on beamline I911-3 at the MAX-II synchrotron, Lund, Sweden. Full details of data collection and processing have been presented in a previous article (Manzoni et al., 2016). In that article, the neutron data set used here was referred to as 'lactose-2' and was $87.1 \%$ complete to $1.7 \AA$ resolution $(66.5 \%$ from 1.8 to $1.7 \AA$ ). The X-ray data are $99 \%$ complete to $1.0 \AA$ resolution. However, in all refinements in this paper the X-ray data were truncated at $1.7 \AA$ resolution. This was mainly performed because the program used for refinement, $n C N S$ (see below), cannot refine anisotropic $B$ factors. However, it also reflects a more common scenario than that which prevails for galectin-3C, namely that the X-ray and neutron diffraction limits are more similar. Furthermore, high-resolution X-ray data contain information on hydrogen positions that is potentially contradictory to the neutron data, as the peaks in the electron density are displaced towards the centre of the bond to the heavy atom, resulting in shorter bond lengths. A scenario with much higher resolution X-ray data was tested for LPMO. The finally published galectin-3C-lactose structure (Manzoni et al., 2018), deposited in the PDB as entry 6eym, was refined against a more complete neutron data set $(96 \%$ 
Table 1

Correspondence between the water molecules discussed in the text and their numbers in the deposited structure (PDB entry 6eym).

\begin{tabular}{ll}
\hline Text & PDB entry 6eym \\
\hline Wat-1 & Wat-648 \\
Wat-2 & Wat-668 \\
Wat-3 & Wat-666 \\
Wat-4 & Wat-614 \\
Wat-5 & - \\
Wat-6 & - \\
Wat-7 & - \\
Wat-8 & Wat-655 \\
\hline
\end{tabular}

complete to $1.7 \AA$ resolution) with higher multiplicity that was obtained by merging the data from two crystals (Manzoni et al., 2016).

The starting structure for refinement was the $0.86 \AA$ resolution X-ray structure of galectin-3 in complex with lactose with the water molecules removed (Saraboji et al., 2012). The structure was first refined against the X-ray data using REFMAC5 (Murshudov et al., 2011). Water molecules were added manually when visible at $1.7 \AA$ resolution. Geometric restraints for the lactose molecule were generated in phenix.elbow. Joint X-ray/neutron refinement was then performed using phenix.refine (Afonine et al., 2012). All calculations were performed on one core of an Intel Xeon E5-2650 processor at the LUNARC Aurora cluster at Lund University.

Upon the convergence of conventional joint refinement, the final structure was refined for 50 steps with the neutron crystallography patch of the Crystallography and NMR System (Brünger et al., 1998) software (nCNS; Adams et al., 2009) before QM refinement. We used the standard $n C N S$ MM

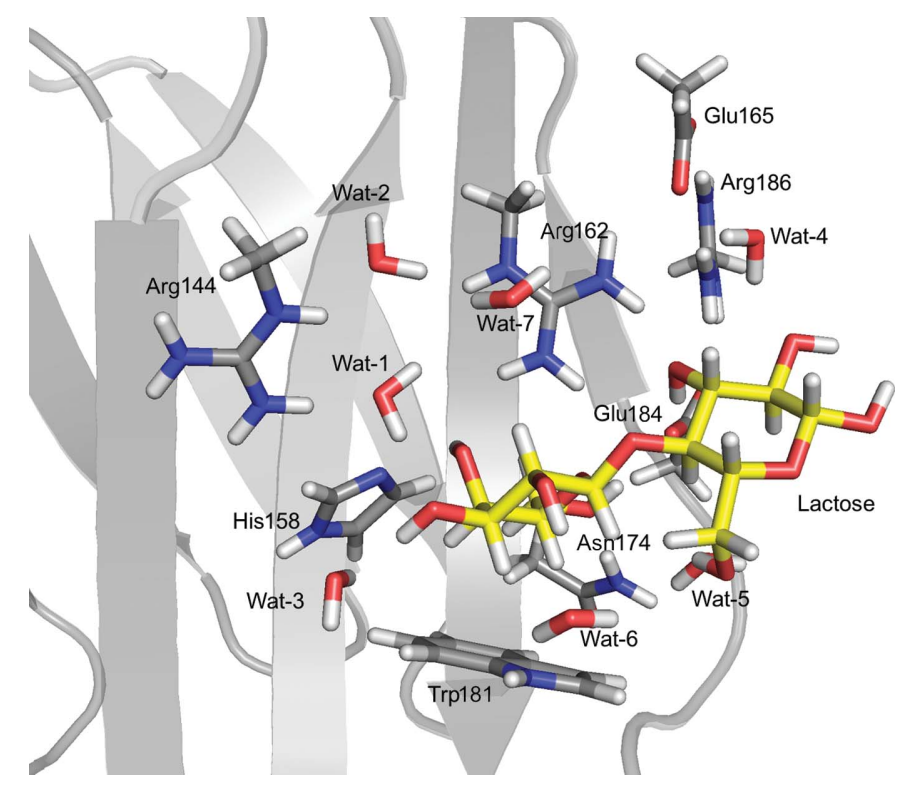

Figure 1

The QM system ( $\mathrm{S} 1$ in Fig. 3) used in the ComQum- $U$ calculations for galectin-3. All atoms involved in the QM calculation are shown as sticks, while the rest of the protein is shown as a cartoon and the remaining water molecules are hidden. Lactose is shown with yellow $\mathrm{C}$ atoms, whereas the protein residues are shown with grey $\mathrm{C}$ atoms. force field for all atoms (protein-allhdg_d.param and dod.param). The atom types and parameters used for lactose are presented in Supplementary Tables S2 and S3 (however, the lactose parameters will not affect the QM-refined results because they cancel exactly, as will be discussed below). After QM refinement, bulk-solvent correction and $B$-factor refinement were performed in PHENIX. Real-space difference density $Z$-scores (RSZDs) were calculated for selected structures with the EDSTATS module (Tickle, 2012) of the CCP4 software suite (Winn et al., 2011).

The QM system for galectin-3 (Fig. 1) consisted of the lactose ligand as well as the side chains of Arg144, His158, Arg162, Glu165, Asn174, Trp181, Glu184 and Arg186, and seven water molecules (named Wat-1, Wat-2, Wat-3, Wat-4, Wat-5, Wat- 6 and Wat-7 in the text; see Table 1 for the corresponding numbers in the deposited structure); 153 atoms in total. The QM system was selected to include all protein residues and water molecules that form hydrogen bonds to the lactose ligand, as well as one residue (Glu-165) and a few water molecules that connect these residues by hydrogen bonds. The Arg, His, Glu, Asn and Trp side chains were truncated in the QM system by replacing the $\mathrm{CG}, \mathrm{CB}, \mathrm{CB}, \mathrm{CA}$ and $\mathrm{CB}$ atoms, respectively, by an $\mathrm{H}$ atom (the hydrogen linkatom approach), as implemented in the ComQum- $U$ software (Ryde, 1996; Ryde \& Olsson, 2001).

\subsection{LPMO}

$\mathrm{X}$-ray structure factors, coordinates, occupancies and $B$ factors were obtained from the $1.1 \AA$ resolution structure of AA10 LPMO (Bacik et al., 2017; PDB entry 5vg0). Neutron structure factors were obtained from PDB entry $5 \mathrm{vg} 1$, a $2.1 \AA$ resolution structure of the same AA10 LPMO (Bacik et al., 2017). Prior to QM refinement, classical X-ray-neutron joint refinement was performed using phenix.refine, starting from the coordinates of the X-ray structure and adding $\mathrm{D}$ atoms with phenix.ready_set. $\mathrm{D}$ atoms at the $\mathrm{N}$-terminal atoms were added manually. Full details of the classical joint refinement can be found in Caldararu et al. (2018). The same QM refinement procedure was then performed as described for galectin-3, except that the X-ray data were not truncated (i.e. all data to $1.1 \AA$ resolution were kept in the re-refinements). However, anisotropic $B$ factors were discarded as $n C N S$ cannot handle these.

The QM system for LPMO (Fig. 2) consisted of the first coordination sphere of the copper ion in either subunit $A$ or $B$ of the enzyme. It contained the copper ion, the imidazole ring of His109, the phenyl ring of Phe164, the full His32 residue, an $\mathrm{O}_{2}$-derived ligand bound to the $\mathrm{Cu}$ atom and a crystal water molecule (Wat-301 in subunit $A$ and Wat-307 in subunit $B$ ). The three residues were truncated in the QM system by replacing the $\mathrm{CB}$ atoms or $\mathrm{C}$ atom (for His32) with $\mathrm{H}$ atoms, as described above. Based on a study of alternative oxidation states (Caldararu et al., 2018), the copper ion was considered to be $\mathrm{Cu}^{2+}$, while the oxygen species was considered to be a peroxide, $\mathrm{O}^{2-}$, resulting in a total net charge on the system of 0 . 


\subsection{QM calculations}

The QM calculations were carried out using TURBOMOLE 7.0 (Furche et al., 2014). The TPSS (Tao et al., 2003) density functional theory (DFT) method and the def2-SV(P) basis set (Schäfer et al., 1992) were used. Dispersion effects were included with the empirical DFT-D3 approach (Grimme et al., 2010). This approach gives reasonable geometries for both biochemical and bioinorganic systems (Neese, 2006; Sure \& Grimme, 2015; Antony et al., 2015) and have been used in many applications in our group (Ryde, 2016).

\section{Result and discussion}

\subsection{Implementation}

The added value of QM calculations for crystallographic structure determination is to make the model consistent with both the crystallographic data and the QM energy function, avoiding the less accurate MM potential, and hence provide a chemically more reasonable model. QM is introduced for a small but interesting part of the structure in the QM/MM approach. Therefore, we introduce QM calculations only in the final steps of the structure-determination process, typically after a standard structure refinement. This will not affect the overall structure radically, but will provide better local geometries, help in resolving ambiguous interpretations of the density maps and support conclusions about unusual geometries.

We decided to implement the combined QM and neutron crystallographic refinement in the $n C N S$ software (Adams et al., 2009) for several reasons. Firstly, $n C N S$ is freely available software, providing implementation of both neutron and joint neutron and X-ray refinement. Secondly, our previous quantum-refinement method was implemented in the CNS software (Brünger et al., 1998), which made the extension to neutron crystallography straightforward. Thirdly, CNS was originally developed from the CHARMM MM software (Brooks et al., 2009), meaning that it consists of an open symbolic language with existing implementation of MM force fields, as well as facile access to and manipulation of energies and forces, again strongly simplifying the implementation.

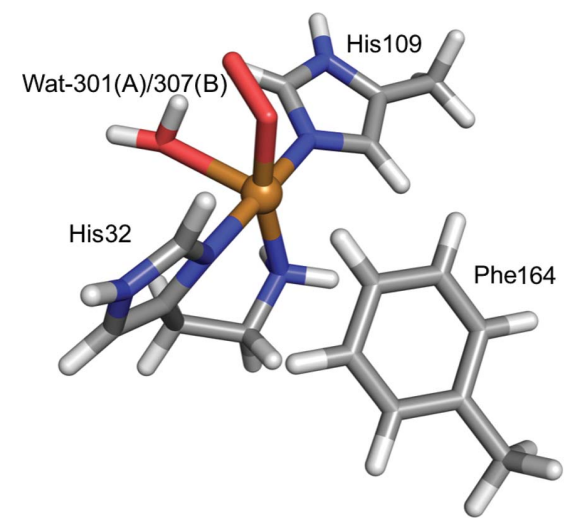

Figure 2

The QM system (S1 in Fig. 3) used in the ComQum-U calculations for LPMO.
Crystallographic refinement is in principle a global pseudoenergy minimization using an energy function of the form

$$
E_{\text {cryst }}=w_{\mathrm{X}} E_{\mathrm{X}-\text { ray }}+w_{\mathrm{N}} E_{\text {neutron }}+E_{\mathrm{MM} 12}
$$

where $E_{\mathrm{MM} 12}$ is an $\mathrm{MM}$ (or another empirical or statistical) energy function of the entire model (the meaning of the ' 12 ' subscript will be apparent below when the total system is divided into two parts), whereas $E_{\mathrm{X} \text {-ray }}$ and $E_{\text {neutron }}$ describe how closely the current model reproduces the experimental X-ray and neutron data, respectively. The latter could, in principle, be the crystallographic $R$ factors, but typically more sophisticated maximum-likelihood refinement target functions are employed (Pannu \& Read, 1996; Adams et al., 1997). The two weight factors $w_{\mathrm{X}}$ and $w_{\mathrm{N}}$ are needed because $E_{\mathrm{MM} 12}$ is typically in energy units, whereas the other two terms are plain numbers. They determine the relative weights of the three (pseudo-)energy terms so that, for example, setting $w_{\mathrm{X}}=0$ implies that a pure neutron crystallographic refinement is performed. Ideal values of the weight factors can be obtained by optimization of the $R_{\text {free }}$ factor, but this is tedious for the joint energy function and therefore they are typically determined so that the three energy terms have a similar magnitude in a short MD simulation (Brünger \& Rice, 1997; Brünger et al., 1989; Adams et al., 1997).

QM calculations can be introduced into this energy function by simply replacing $E_{\mathrm{MM} 12}$ with a standard QM/MM energy function (Ryde, 2016). In this, QM is employed for a restricted, but interesting, part of the protein, called system 1 or the QM system. MM is employed for the remaining atoms in the considered model, called system 2 (and therefore the entire system will be called 12 , as in $\left.E_{\mathrm{MM} 12}\right)$. Within the subtractive QM/MM approach (Cao \& Ryde, 2018), this is obtained in the following way:

$$
E_{\mathrm{QM} / \mathrm{MM}}=E_{\mathrm{QM} 1}+w_{\mathrm{MM}}\left(E_{\mathrm{MM} 12}-E_{\mathrm{MM} 1}\right) .
$$

Here, $E_{\mathrm{QM} 1}$ is the QM energy of system 1 and $E_{\mathrm{MM} 1}$ is the MM energy of the same system. The latter ensures that no energy terms are double-counted by cancelling the MM terms of the QM system in $E_{\mathrm{MM} 12}$ (therefore, the results will not depend on the MM parameters employed for the QM system). $w_{\mathrm{MM}}$ is a scale factor, which is needed because the statisticsbased force field in CNS (Engh \& Huber, 1991) typically gives energies that are approximately three times larger than an energy-based force field (Ryde et al., 2002). Therefore, $w_{\mathrm{MM}}$ was always set to $1 / 3$. In standard QM/MM with energy-based force fields, $w_{\mathrm{MM}}=1$ and is therefore normally omitted (Ryde, 2016).

Replacing $E_{\mathrm{MM} 12}$ in (1) with $E_{\mathrm{QM} / \mathrm{MM}}$ in (2) gives the final combined energy function:

$$
\begin{aligned}
E_{\text {ComQum- } U}= & w_{\mathrm{X}} E_{\mathrm{X}-\text { ray }}+w_{\mathrm{N}} E_{\text {neutron }}+E_{\mathrm{QM} 1} \\
& +w_{\mathrm{MM}}\left(E_{\mathrm{MM} 12}-E_{\mathrm{MM} 1}\right) .
\end{aligned}
$$

Forces were obtained from this energy function by using analytical differentiation and employing the chain rule for the hydrogen link-atoms (Ryde, 1996; Ryde \& Olsson, 2001). 
The flow chart of the ComQum- $U$ program is shown in Fig. 3. It is implemented using the $n C N S$ xn_minimize. inp and xn_bindividual. inp files, with some simple modifications to write out crystallographic energies and forces. This ensures that all normal crystallographic manipulations and calculations are performed, for example bulk-solvent corrections and calculations of $R$ factors. Moreover, it was necessary to read in and write out coordinates with a higher precision than standard PDB files to avoid convergence problems (Ryde et al., 2002). For calculation of the crystallographic energies and forces the number of minimization steps was set to zero, whereas in the relaxation step it was set to one. The maximumlikelihood refinement target using amplitudes was employed. A simple $C N S$ script was also employed to calculate the $E_{\mathrm{MM} 1}$ energy term. The whole quantum-refinement procedure is driven by a Linux shell script, which is based on the TURBOMOLE geometry-optimization script jobex (Furche et al., 2014). Relaxation of the QM system is performed by the relax program in TURBOMOLE, employing a BroydenFletcher-Goldfarb-Shanno quasi-Newton approach. Geometry optimizations were continued until the energy change between two iterations was less than $2.6 \mathrm{~J} \mathrm{~mol}^{-1}\left(10^{-6}\right.$ arbitrary units) and the maximum norm of the Cartesian gradients was below $10^{-3}$ arbitrary units. A further description of the procedure can be found at http://signe.teokem.lu.se/ ulf/ Methods/comqum_u.html, and the interface can be provided by the authors upon request (but note that $n C N S$ and TURBOMOLE need to be installed separately and that a licence for TURBOMOLE is required). A typical re-refinement of the galectin-3 test system required around $150 \mathrm{QM}$ energy and gradient calculations, which took about $5 \mathrm{~h}$ on a single processor, whereas re-refinement of the smaller LPMO test system required only around $90 \mathrm{QM}$ energy and gradient calculations, which took about $2 \mathrm{~h}$ on a single processor.

Standard crystallographic refinement is performed without any electrostatic MM term. For X-ray data this is natural because the positions of the $\mathrm{H}$ atoms are normally unknown and the electrostatics of hydrogen bonds strongly depend on the hydrogen positions. For neutron structures, the positions of most (but typically not all) H (D) atoms are known, but the refinement is still traditionally performed without any electrostatics. To make the results compatible with the data

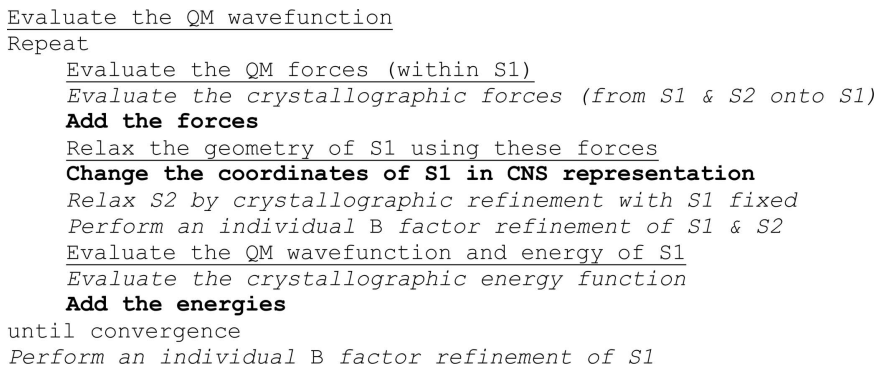

Flow chart of the ComQum- $U$ program. S1 and S2 denotes systems 1 and 2. Steps in bold constitute the actual ComQum-U interface. Steps in italics are performed by the crystallographic refinement program ( $n C N S)$, whereas those that are underlined are run by the QM program. The whole procedure is driven by a Linux shell script. obtained with standard refinement, we decided to exclude electrostatics from the calculations. However, it should be noted that electrostatics can easily be included in the energy function using $\mathrm{MM}$ atomic charges in $E_{\mathrm{MM} 12}$ and a pointcharge model of the protein in $E_{\mathrm{QM} 1}$. The effect of electrostatics will be evaluated in a future study. Finally, it should be noted that QM calculations naturally involve electrostatics and the positions of all $\mathrm{H}$ atoms within the QM system need to be included.

\subsection{Performance on galectin- 3}

The performance of the ComQum- $U$ program was examined by re-refining a preliminary, unpublished $1.7 \AA$ resolution neutron structure of lactose bound to the perdeuterated carbohydrate-recognition domain of galectin-3. The structure was originally refined jointly against X-ray and neutron data from the same crystal to $1.7 \AA$ resolution using phenix.refine (Afonine et al., 2012) and was briefly re-refined with $n C N S$ (Adams et al., 2009) before running the quantum refinement. We included the lactose ligand, eight nearby amino-acid side chains and seven water molecules in the QM system, as shown in Fig. 1 (a total of 153 atoms).

We first checked that the program worked properly by refining the structure with varying values of the weight factors $w_{\mathrm{X}}$ and $w_{\mathrm{N}}$ in (1)-(3) from 0 to 10 . By setting $w_{\mathrm{X}}=w_{\mathrm{N}}=0$, we essentially obtain a $\mathrm{QM} / \mathrm{MM}$ structure of the active site, although with the CNS MM force field and without any electrostatics. This gives a rather poor structure in terms of the crystallographic $R$ factors $(0.25 / 0.23,0.21 / 0.19$; these four values are $R_{\text {free }} / R$ first for the neutron data and then for the $\mathrm{X}$-ray data).

Next, we performed refinements with $8 \times 8$ different values of the weight factors $w_{\mathrm{X}}$ and $w_{\mathrm{N}}(0,0.01,0.03,0.1,0.3,1,3$ and $10)$. The resulting $R$ values are shown in Fig. 4 and Supplementary Table $\mathrm{S} 1$. It can be seen that the best $R$ value for the neutron data (0.214) was obtained for the largest $w_{\mathrm{N}}=10$, as expected. It increased slightly when $w_{\mathrm{X}}$ was increased. Likewise, the best $R$ value for the X-ray data (0.179) was obtained for the largest $w_{\mathrm{X}}=10$. However, the best values of $R_{\text {free }}$ for both the neutron and X-ray data ( 0.233 and 0.207 , respectively) are obtained at intermediate values at around $w_{\mathrm{N}}=1$ and $w_{\mathrm{X}}=3$. This shows that the refinement function works properly and that optimum values of the two weight factors can be obtained by optimizing the two $R_{\text {free }}$ values, although the surfaces are quite flat. Performing an additional bulksolvent correction and $B$-factor refinement in PHENIX decreased the $R_{\text {free }}$ values to 0.179 for the X-ray data and 0.215 for the neutron data (Table 2), which is comparable to the value of 0.211 obtained from phenix.refine alone.

In Fig. 5, we compare structures refined without QM, as well as with QM with the optimum weights $w_{\mathrm{N}}=1$ and $w_{\mathrm{X}}=3$. It can be seen that the largest movements are observed for the $\mathrm{D}$ atoms, in particular those of water molecules, whereas the heavy atoms typically remain at the same positions.

For example, one of the largest changes is observed for the weakly bound water molecule 5 , which shows quite unusual 
hydrogen bonds in the original structure, with one $\mathrm{D}$ atom directed towards the side-chain DB atom of Trp181 (2.26 $\AA$ ) and the other $\mathrm{D}$ atom pointing towards the solvent (Fig. $6 a$ ). In the QM-refined structure, this water molecule rotates by $90^{\circ}$ so that the deuterons are directed towards the $\mathrm{O} 6$ atom of lactose $(2.39 \AA)$ and one of the OE atoms of Glu184 (2.39 $\AA$ ). In both cases, the water molecule also receives a hydrogen bond from the $\mathrm{DO}^{\prime}$ atom of lactose (2.11 and $2.27 \AA$, respectively).

Wat- 6 is also weakly bound and shows large changes in the positions of the D atoms (Fig. $6 b$ ). In the original structure it does not form any hydrogen bonds. Instead, one of the deuterons is only $2.48 \AA$ from DE1 of Trp181, in a seemingly unfavourable interaction. In the QM-refined structures Wat-6 rotates so that it donates a hydrogen bond to $\mathrm{O6}^{\prime}$ of lactose. In both cases, it also receives a hydrogen bond from the DO2 atom of lactose, but this interaction is also significantly improved in the QM-refined structure, with a $\mathrm{D}-\mathrm{O}$ distance of $1.80 \AA$ compared with $2.06 \AA$.

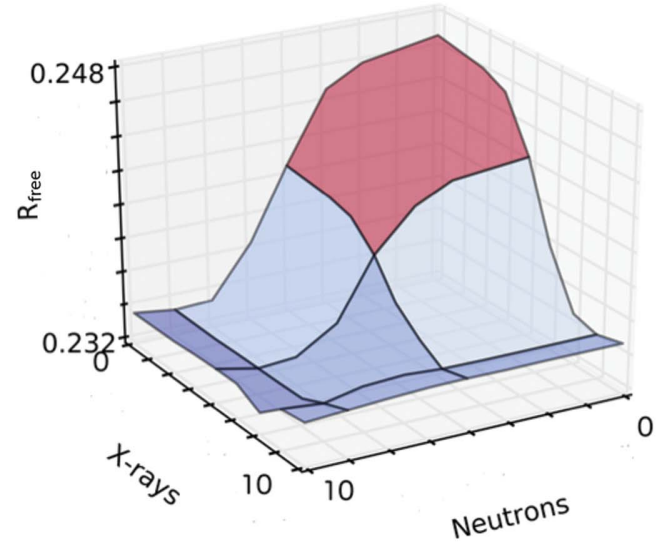

(a)

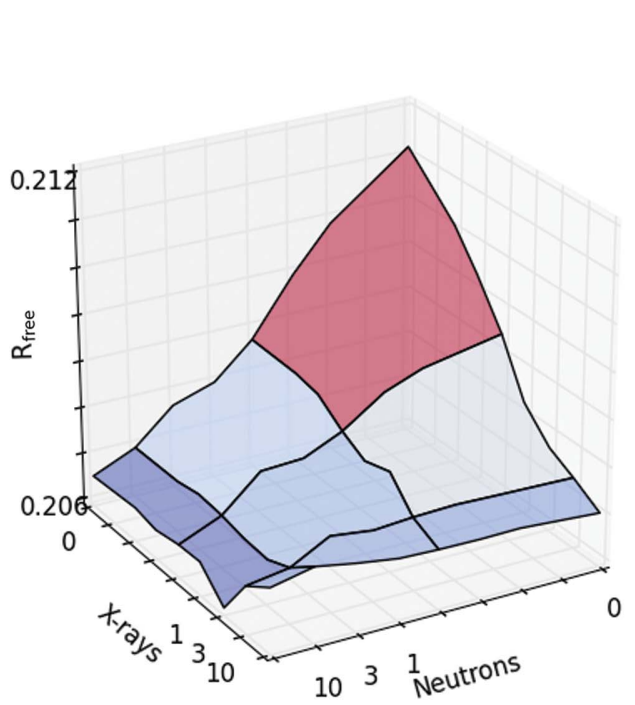

(c)

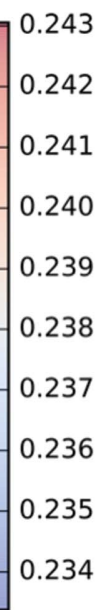

0.242

0.241

0.240

0.239

0.238

0.237

0.236

0.235

0.234

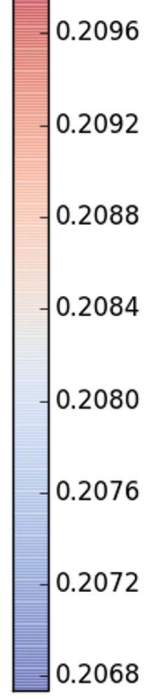

Figure 4

The dependence of the $R$ factors on the weight factors $w_{\mathrm{N}}$ and $w_{\mathrm{X}}$ in the refinement of galectin-3 (see equation 3$):(a) R_{\text {free}}^{\mathrm{N}},(b) R^{\mathrm{N}},(c) R_{\mathrm{free}}^{\mathrm{X}}$ and $(d) R^{\mathrm{X}}$. The raw data are given in Supplementary Table S1.
Wat-7 also shows a rather large reorganization (Fig. 6c). In the original structure one of the $\mathrm{D}$ atoms points towards the $\mathrm{D}$ atom of another water molecule (Wat-2, 1.69 A). In the QMrefined structure this $\mathrm{D}$ atom instead points towards lactose (but without forming any particular hydrogen bond) so that the $\mathrm{O}$ atom can receive a strong hydrogen bond from Wat-2 (D-O distance of $1.72 \AA$ ).

Finally, Wat-4 also experiences a reorganization of the hydrogen-bond network (Fig. 6d). In the original structure one of the $\mathrm{D}$ atoms points towards the solution. However, in the QM-refined structure it instead forms a strong hydrogen bond to the OE2 atom of Glu-165 (1.84 $\AA$ ). In both cases the other $\mathrm{D}$ atom forms a hydrogen bond to $\mathrm{O} 2^{\prime}$ of lactose, although it is shortened by $0.1 \AA$ in the QM/MM structure (from 1.99 to $1.86 \AA$ ). Thereby, it bridges the interaction between Glu-165 and the ligand.

In the ligand, the $\mathrm{DO}^{\prime}$ atom shows the largest movement, with an $\sim 100^{\circ}$ change in the $\mathrm{DO} 1^{\prime}-\mathrm{O}^{\prime}-\mathrm{C1}^{\prime}-\mathrm{C}^{\prime}$ dihedral angle (Fig. 5). This is probably to relieve a short contact

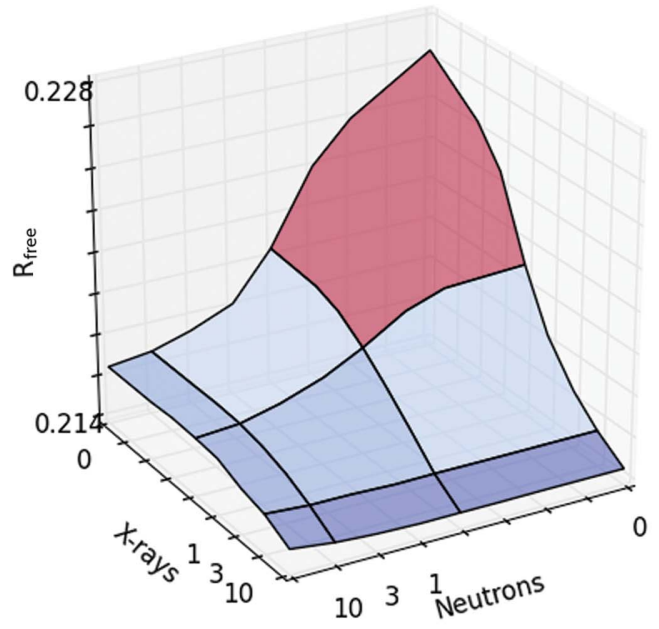

(b)

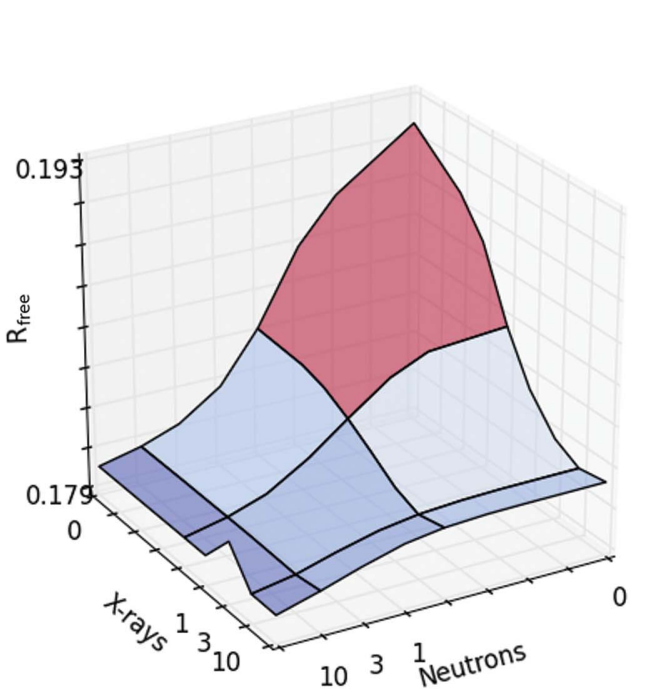

0.1864

0.1856

0.1848

$-0.1840$

0.1832

0.1824

$-0.1816$

0.1808

0.1800

(d) 
Table 2

Structure quality of the two galectin-3 structures before and after QM refinement, with $w_{\mathrm{N}}=1$ and $w_{\mathrm{X}}=3$ for the preliminary structure and $w_{\mathrm{N}}=7$ and $w_{\mathrm{X}}=7$ for the deposited structure.

$R_{\text {work }}$ and $R_{\text {free }}$ were calculated in $n C N S$. Geometry statistics were calculated with MolProbity.

\begin{tabular}{|c|c|c|c|c|}
\hline & \multicolumn{2}{|c|}{ Preliminary } & \multicolumn{2}{|c|}{ Deposited } \\
\hline & Before & After & Before & After \\
\hline$R_{\text {work }}(\mathrm{X}$-ray $)$ & 0.181 & 0.179 & 0.189 & 0.189 \\
\hline$R_{\text {free }}(\mathrm{X}$-ray $)$ & 0.208 & 0.207 & 0.195 & 0.195 \\
\hline$R_{\text {work }}$ (neutron) & 0.221 & 0.216 & 0.219 & 0.218 \\
\hline$R_{\text {free }}$ (neutron) & 0.238 & 0.233 & 0.221 & 0.221 \\
\hline R.m.s.d., bonds $(\AA)$ & 0.021 & 0.022 & 0.011 & 0.018 \\
\hline R.m.s.d., angles $\left({ }^{\circ}\right)$ & 1.9 & 1.9 & 1.7 & 1.7 \\
\hline Ramachandran favoured (\%) & 98.5 & 98.5 & 97.1 & 98.5 \\
\hline Ramachandran allowed (\%) & 3.3 & 1.5 & 2.9 & 1.5 \\
\hline Ramachandran outliers (\%) & 0.0 & 0.0 & 0.0 & 0.0 \\
\hline Rotamer outliers (\%) & 0.8 & 0.8 & 0.0 & 0.0 \\
\hline All-atom clashscore & 4.87 & 3.10 & 4.00 & 1.77 \\
\hline
\end{tabular}

between $\mathrm{DO}^{\prime}$ and $\mathrm{DO} 2^{\prime}(2.05 \AA)$. However, in neither the original structure nor in the QM-refined structure does $\mathrm{DO}^{\prime}$ form any hydrogen bond to the surroundings (it is solventexposed, but there are no visible water molecules close to it). The movement of $\mathrm{DO}^{\prime}$ is negligible.

The other atoms in both the ligand and the protein show only smaller adjustments within the same local minimum, typically to improve hydrogen-bond interactions. For example, DO4 in lactose moves by $0.70 \AA$ to shorten the hydrogen-bond distance to NE2 of His158 from 2.16 to $1.68 \AA$ (Fig. 5). The largest movement of a protein atom is that of DH12 of Arg144, which moves to improve the hydrogen bond to a water molecule (Wat-1) from 1.77 to $1.62 \AA$, as shown in Fig. 6(e). On average, the protein atoms in the QM system move by only $0.06-0.17 \AA$, again with the largest movement for Arg144.

If we instead compare the best QM-refined structure with a pure $\mathrm{QM} / \mathrm{MM}$ structure, obtained with $w_{\mathrm{N}}=w_{\mathrm{X}}=0$, much

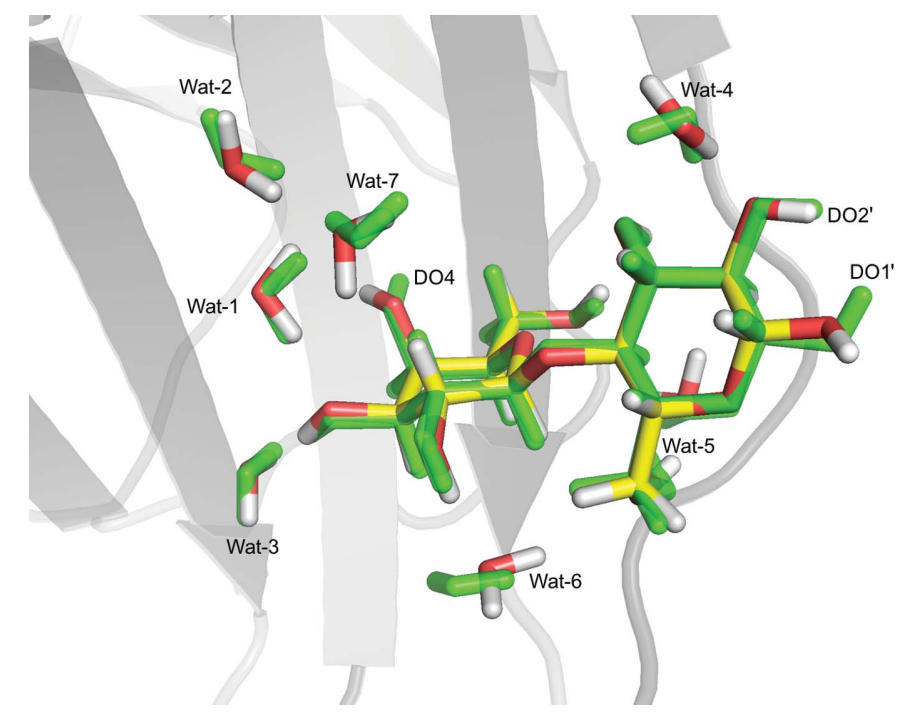

Figure 5

Structure before (green) and after (C atoms in yellow) QM refinement of the preliminary galectin-3 structure with $w_{\mathrm{N}}=1$ and $w_{\mathrm{X}}=3$. Protein residues are hidden for clarity (they basically do not move). larger movements are observed, as can be seen in Fig. 7. In fact, the median movement of the atoms in the QM system is $0.5 \AA$, compared with $0.1 \AA$ for the QM-refined structure. For most residues this represents a slight change (rotation or tilt) in the orientation of the amino-acid side chains. However, several water molecules move by up to $1.8 \AA$ and several lactose atoms also move significantly (up to $1.7 \AA$ for $\mathrm{O}^{\prime}$ and $\left.\mathrm{DO}^{\prime}\right)$. This larger movement of course reflects that lactose and water are not as restrained by the surrounding structure as the amino-acid side chains, which are kept in place by the backbone atoms (which were kept fixed in the optimization). However, as can be seen from the figure, the water molecules move away from the electron and neutron densities, illustrating the advantage of the joint $\mathrm{QM}$ refinement. The discrepancy between the $\mathrm{QM} / \mathrm{MM}$ and experimental data is caused by the fact that the latter include dynamics and entropy, as well as the averaged effects of the surrounding solvent, but also disorder in the crystal.

Next, we studied the deposited neutron structure of lactose bound to galectin 3 (PDB entry 6eym), which has a higher completeness of the neutron data and was previously jointrefined in PHENIX 1.12. Compared with the preliminary structure, the deposited structure contains fewer water molecules around the lactose molecule (Fig. 8). Interestingly, three of the four water molecules (Wat-5, Wat-6 and Wat-7) that showed large reorganization after quantum refinement are absent in the more conservatively interpreted deposited structure, suggesting that these water molecules were not well ordered in the original structure. Nuclear difference density for these waters can be seen in PDB entry 6eym at low contour levels. Moreover, Wat-4 in the deposited structure is in the orientation suggested by quantum refinement of the first structure, proving that QM refinement of the preliminary structure found the correct orientation of this water molecule.

We also performed quantum refinement of the deposited structure, including only five water molecules in the QM system: Wat-1, Wat-2, Wat-3, Wat-4 and Wat-8 (see Table 1 for the corresponding water numbers in the deposited structure, PDB entry 6eym). Wat-8, which was not present in the preliminary structure, forms hydrogen bonds to Wat-3 and lies close to Trp181. The lactose molecule also shows a different orientation of two D atoms, DO4 and DO2'. Strikingly, in the deposited structure the DO4 atom is close to the position in the quantum refinement of the preliminary structure. No protein residues show any significant reorientation between the two structures.

The joint QM refinement of the deposited structure, using the optimized weights from the previous $\mathrm{QM}$ refinement, $w_{\mathrm{N}}=1$ and $w_{\mathrm{X}}=3$, gave an $R_{\text {free }}$ of 0.211 for the neutron data and 0.156 for the X-ray data (after bulk-solvent correction and $B$-factor refinement in PHENIX). However, with these weights several atoms move out of the nuclear density after quantum refinement, for example Wat-1, Wat-2 and the ligand DO2 atom (Fig. 9a). Therefore, we performed two sets of weight searches starting from two relative weights: $w_{X} / w_{\mathrm{N}}=3$, which were the weights used in the preliminary structure, and $w_{\mathrm{X}} / w_{\mathrm{N}}=1$ as used in Manzoni et al. (2018). We used a linear 


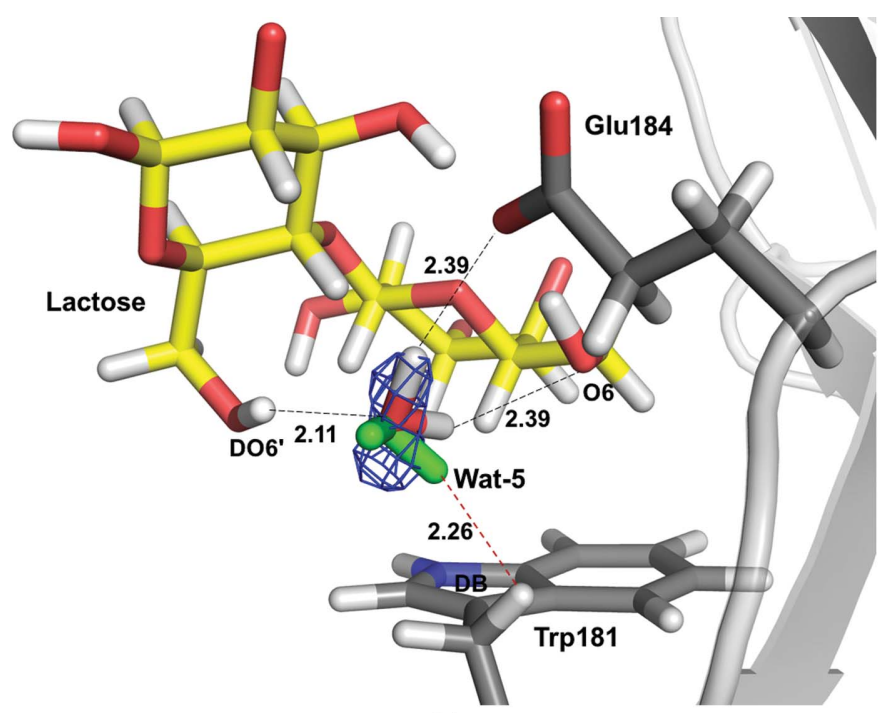

(a)

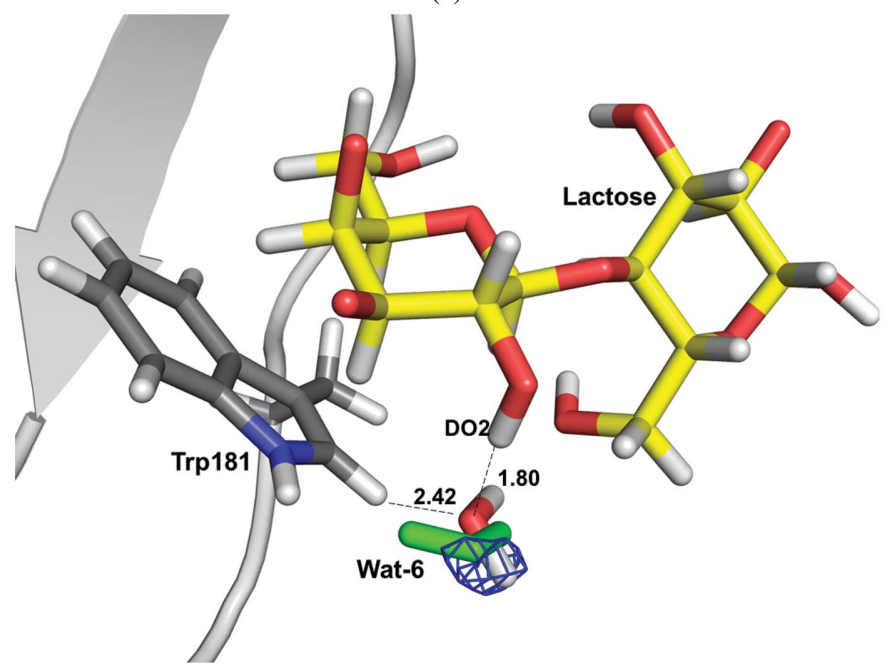

(b)

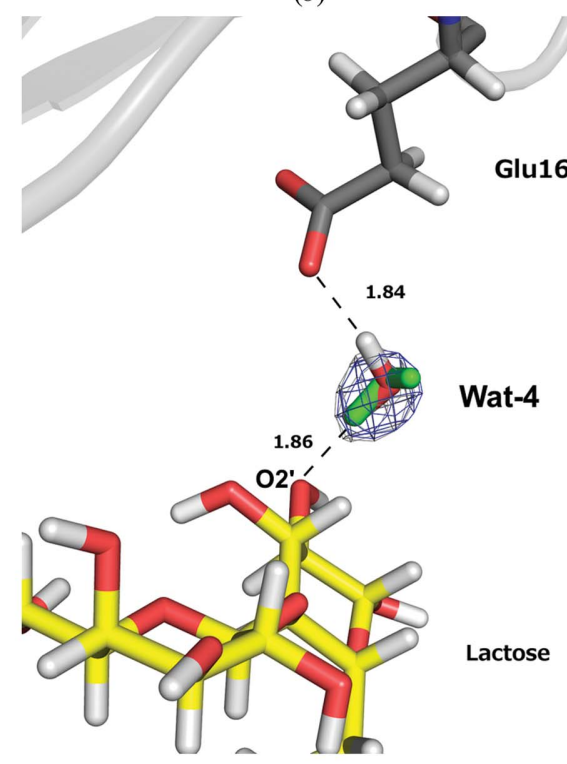

(d) search from $w_{\mathrm{N}}=w_{\mathrm{X}}=1$ to $w_{\mathrm{N}}=w_{\mathrm{X}}=10$ and from $w_{\mathrm{N}}=1$ and $w_{\mathrm{X}}=3$ to $w_{\mathrm{N}}=10$ and $w_{\mathrm{X}}=30$, respectively. In order to compare the quality of the resulting structures, we calculated the maximum absolute RSZD values of lactose and water molecules from the neutron maps, which measure the local accuracy of the model, being sensitive to local discrepancies. As can be seen in Table 3, the structure obtained from QM refinement with $w_{\mathrm{N}}=w_{\mathrm{X}}=7$ fits the crystallographic data best, with the RSZDs of Wat-1 and the lactose molecule being over one unit lower than in QM refinements using lower weights. However, other weights can be used to obtain structures of similar quality, for example structures obtained with $w_{\mathrm{N}}=$ $w_{\mathrm{X}}=6$ or $w_{\mathrm{N}}=5$ and $w_{\mathrm{X}}=15$ give rise to similar RSZD values of the residues in the quantum system. The $R_{\text {free }}$ values of the resulting structures do not change compared with those obtained from the QM refinement with $w_{\mathrm{N}}=1$ and $w_{\mathrm{X}}=3$

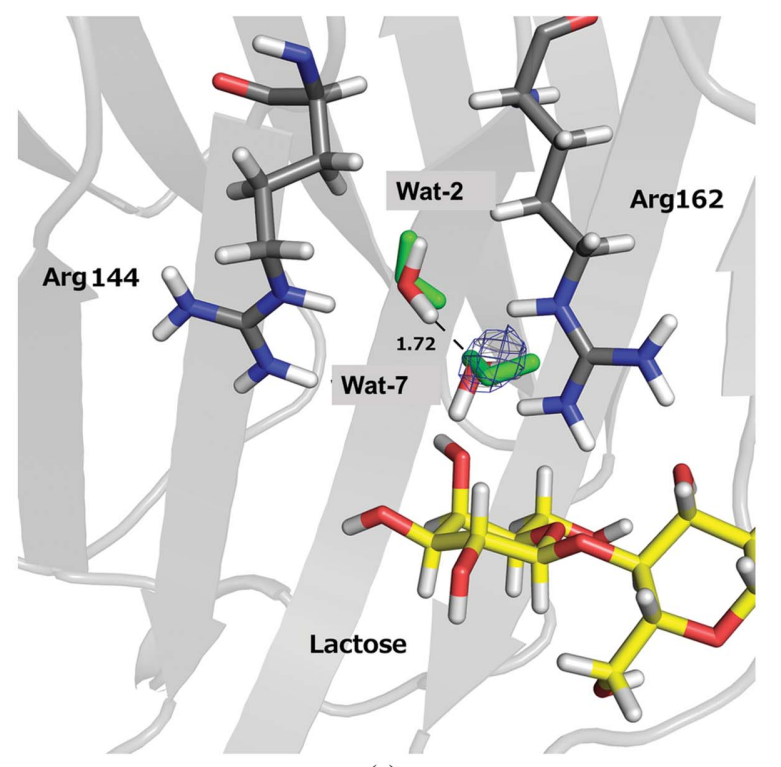

(c)

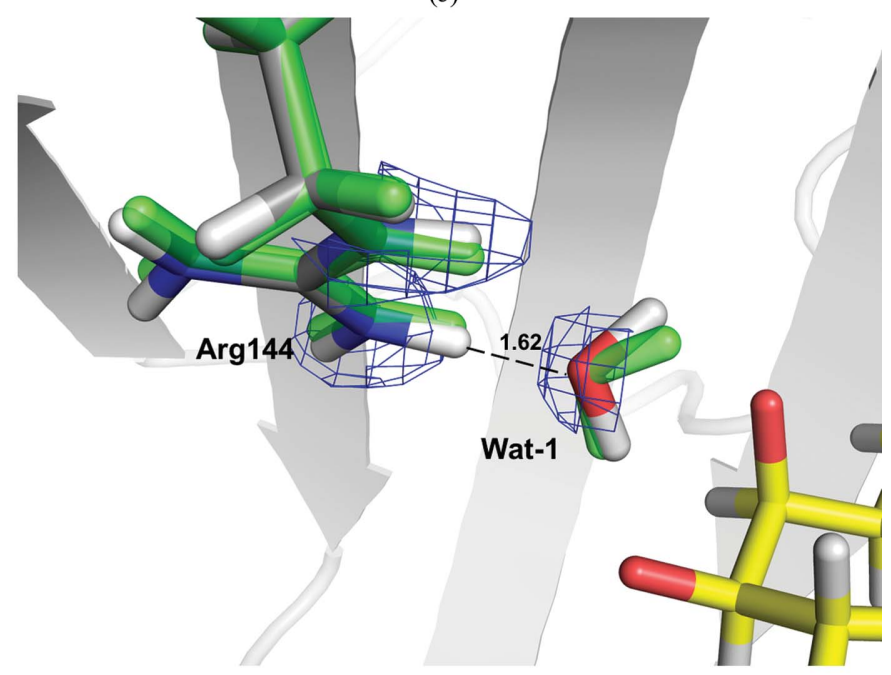

(e)

Figure 6

Significant movement of four water molecules and one protein residue in the QM refinement of the preliminary neutron structure of galectin-3: Wat-5 $(a)$, Wat-6 $(b)$, Wat-7 (c), Wat-4 $(d)$ and Arg144 (e). Atoms are shown in green before and red/white after QM refinement. The nuclear $2 m\left|F_{\mathrm{o}}\right|-D\left|F_{\mathrm{c}}\right|$ density is shown in light blue at $1.0 \sigma$ and in dark blue at $0.7 \sigma$ (for the less well ordered water molecules Wat-5 and Wat-6). 
(0.213 and 0.159; Table 2). For the higher values of the two weights the improvement in the RSZD scores is small and for weights of higher than 10 convergence problems appeared in the QM calculations.

Fig. 9(b) shows a comparison between the original deposited structure and the QM-refined structure with $w_{\mathrm{N}}=w_{\mathrm{X}}=7$ (virtually identical results were also obtained with $w_{\mathrm{N}}=5$ and $\left.w_{\mathrm{X}}=15\right)$. As in the QM refinement of the preliminary structure, the largest movements are observed for D atoms, although to a smaller degree, as the neutron data are of higher quality than in the preliminary structure. The water molecules still change their orientation to form more favourable

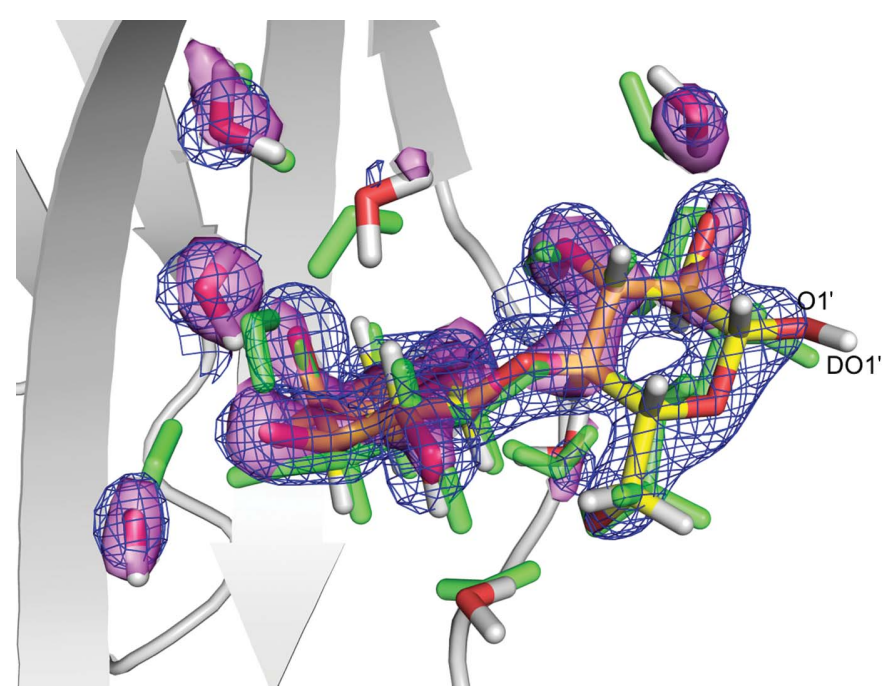

Figure 7

Positions of lactose and the water molecules in QM/MM structures optimized without $\left(w_{\mathrm{X}}=w_{\mathrm{N}}=0\right.$, green $)$ or with restraints to the crystallographic data $\left(w_{\mathrm{N}}=1, w_{\mathrm{X}}=3\right)$. The $2 m\left|F_{\mathrm{o}}\right|-D\left|F_{\mathrm{c}}\right|$ electron density at $1.0 \sigma$ is shown as a blue grid and the nuclear $2 m\left|F_{\mathrm{o}}\right|-D\left|F_{\mathrm{c}}\right|$ density at $1.0 \sigma$ is shown as a violet surface.

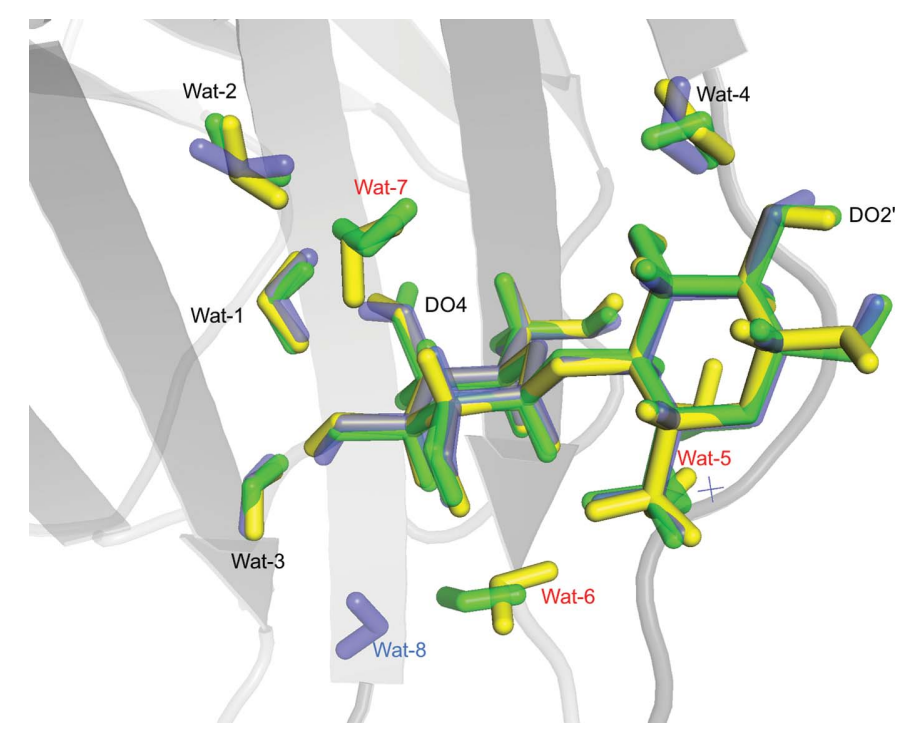

Figure 8

Overlay of the deposited galectin-3 structure (PDB entry 6eym, blue; Manzoni et al., 2018) and the preliminary structure before (green) and after (yellow) QM refinement. Protein residues are hidden for clarity.
Table 3

Maximum absolute RSZD of the lactose molecule and of the five water molecules included in the QM refinement of the deposited galectin-3 structure obtained with different weights of the experimental data $\left(w_{\mathrm{N}}\right.$ and $\left.w_{\mathrm{X}}\right)$.

Note that $w_{\mathrm{X}} / w_{\mathrm{N}}=1$ (left part of table) or 3 (right part).

\begin{tabular}{lllllllllllllll}
\hline$w_{\mathrm{N}}$ & 1 & 2 & 3 & 4 & 5 & 6 & 7 & 8 & 9 & 10 & 1 & 2 & 5 & 10 \\
\hline$w_{\mathrm{X}}$ & 1 & 2 & 3 & 4 & 5 & 6 & 7 & 8 & 9 & 10 & 3 & 6 & 15 & 30 \\
\hline
\end{tabular}

$\begin{array}{lllllllllllllll}\text { Lactose } & 0.6 & 0.8 & 0.8 & 0.4 & 0.3 & 0.3 & 0.2 & 0.8 & 0.2 & 2.8 & 1.5 & 1.6 & 0.2 & 0.2\end{array}$ $\begin{array}{lllllllllllllll}\text { Wat-1 } & 1.5 & 1.5 & 1.2 & 0.6 & 0.4 & 0.1 & 0.0 & 1.6 & 0.1 & 0.1 & 1.4 & 1.1 & 0.1 & 0.1\end{array}$

$\begin{array}{lllllllllllllll}\text { Wat-2 } & 0.9 & 0.9 & 0.9 & 0.6 & 0.8 & 0.7 & 0.7 & 0.9 & 0.9 & 1.0 & 1.0 & 0.9 & 1.0 & 1.4\end{array}$

$\begin{array}{llllllllllllllll}\text { Wat-3 } & 1.2 & 0.8 & 0.8 & 0.9 & 0.8 & 0.8 & 0.8 & 0.9 & 0.8 & 0.7 & 0.9 & 1.0 & 0.9 & 0.8 \\ \text { Wat-4 } & 1.9 & 1.6 & 1.5 & 1.3 & 1 . & 0.9 & 0.8 & 1.6 & 0.6 & 0.6 & 1.1 & 0.9 & 0.6 & 0.5\end{array}$

$\begin{array}{lllllllllllllll}\text { Wat-4 } & 1.9 & 1.6 & 1.5 & 1.3 & 1.1 & 0.9 & 0.8 & 1.6 & 0.6 & 0.6 & 1.1 & 0.9 & 0.6 & 0.5\end{array}$

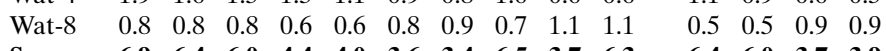

$\begin{array}{lllllllllllllll}\text { Sum } & 6.9 & 6.4 & 6.0 & 4.4 & 4.0 & 3.6 & 3.4 & 6.5 & 3.7 & 6.3 & 6.4 & 6.0 & 3.7 & 3.9\end{array}$

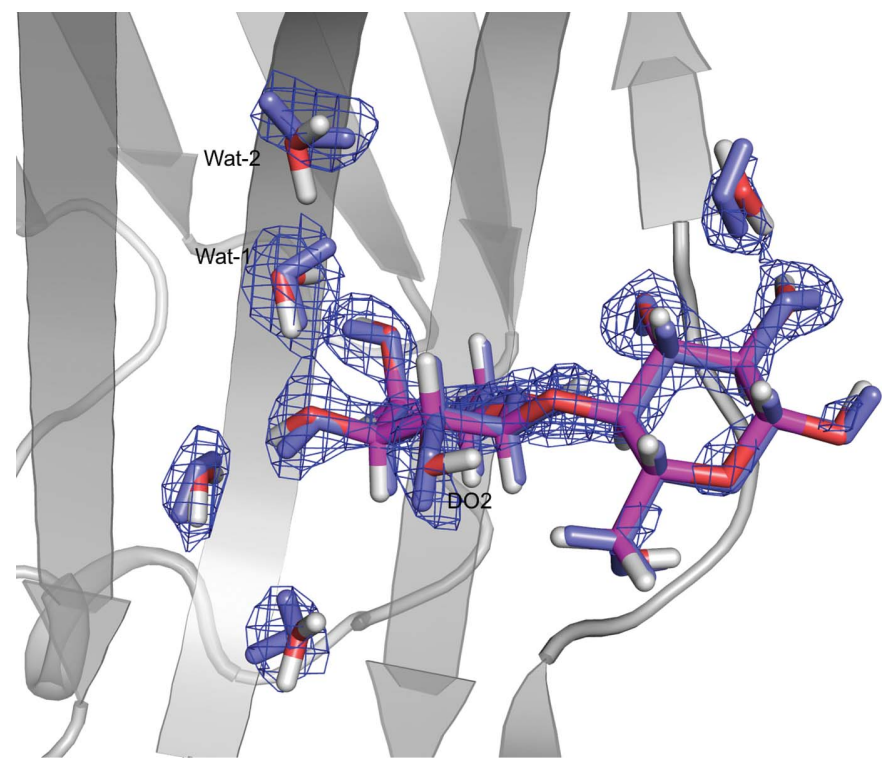

(a)

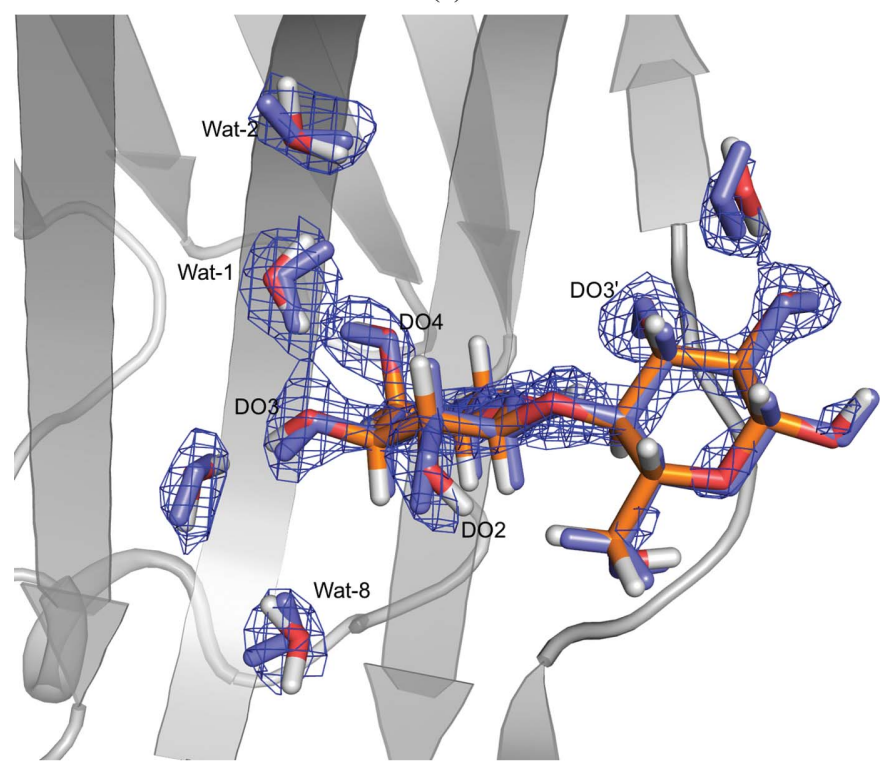

(b)

Figure 9

Overlay of the deposited galectin-3 structure before (blue) and after QM refinement with $(a) w_{\mathrm{X}}=3$ and $w_{\mathrm{N}}=1$ (C atoms in magenta) and $(b) w_{\mathrm{X}}=$ $w_{\mathrm{N}}=7$ (C atoms in orange). The nuclear $2 m\left|F_{\mathrm{o}}\right|-D\left|F_{\mathrm{c}}\right|$ density at $0.8 \sigma$ is shown in blue. Protein residues are hidden for clarity. 
hydrogen bonds, suggesting that QM refinement can also improve structures of high quality.

One such movement is observed for Wat-2. The QM refinement orients its $\mathrm{O}$ atom to form a hydrogen bond to Arg-144 (Fig. 10a), as in the QM refinement of the preliminary structure. The new water molecule introduced into the QM system (Wat-8) also rotates slightly so that one D atom now points towards solution.

In the ligand, several $\mathrm{D}$ atoms show small adjustments to form more favourable hydrogen bonds: DO3 to Wat-3, DO4 to His158 and DO3' to Glu184. The QM refinement is particularly important for the conformation of the DO4 atom, as the nuclear density does not permit an unambiguous assignment of its position (Fig. 10b). DO2 shows the largest movement, but it does not form a hydrogen bond, as the water molecule that it was hydrogen-bonded to in the preliminary structure (Wat-6) is not present in the deposited structure. The protein atoms do not show any significant changes in their position, as in the first QM refinement.

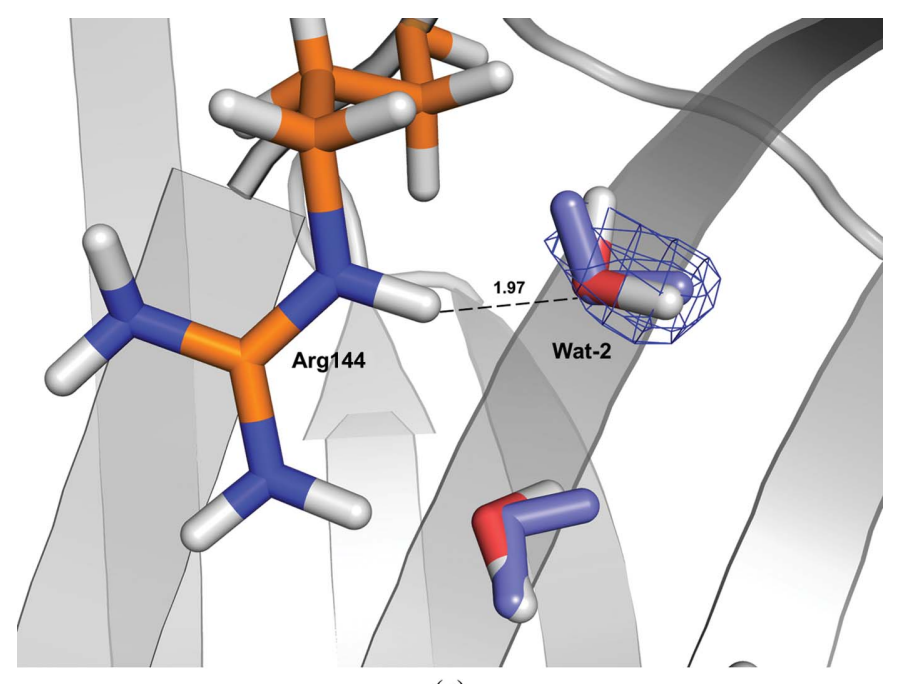

(a)

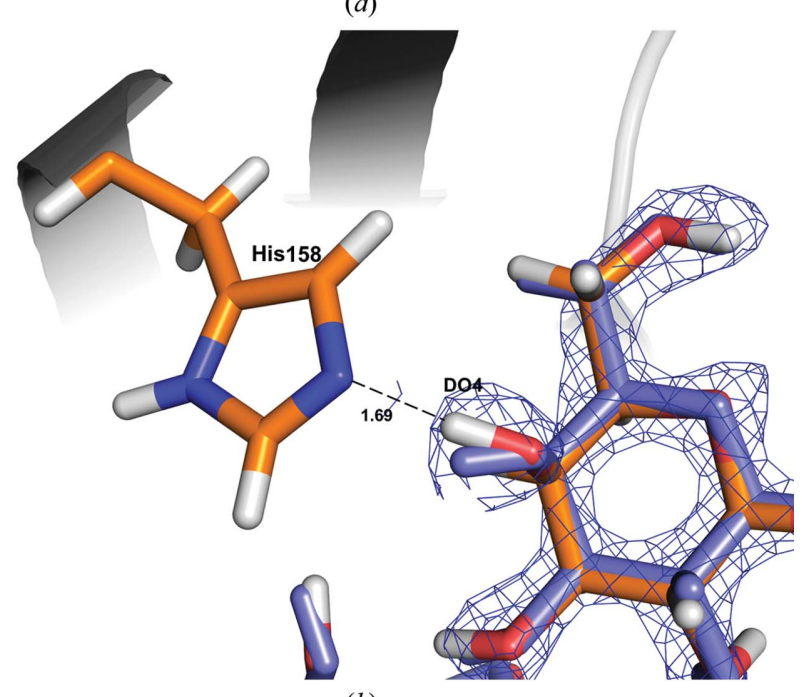

(b)

Figure 10

Movement of water molecule Wat2 $(a)$ and of the DO4 atom in the lactose molecule $(b)$ in order to form more favourable hydrogen bonds after QM refinement of the deposited structure with $w_{\mathrm{X}}=w_{\mathrm{N}}=7$. The nuclear $2 m\left|F_{\mathrm{o}}\right|-D\left|F_{\mathrm{c}}\right|$ density at $1.0 \sigma$ is shown in blue.
To check that the QM refinement had not deteriorated the protein structure, validation statistics were calculated with MolProbity for the final structures $\left(w_{\mathrm{N}}=1\right.$ and $w_{\mathrm{X}}=3$ for the preliminary structure and $w_{\mathrm{N}}=w_{\mathrm{X}}=7$ for the deposited structure). As can be seen in Table 2, the structures show the same quality as before QM refinement, whereas the $R / R_{\text {free }}$ values show a small improvement when compared with the $R / R_{\text {free }}$ values obtained from the standard re-refinement performed in $n C N S$.

Finally, we checked that the results are not sensitive to the starting coordinates by randomly modifying the coordinates by up to $0.1 \AA$ (using phenix.pdbtools) in the QM system and reperforming the QM refinement. This led to the same optimum structure (r.m.s.d. difference of the QM system of $0.05 \AA$ ). Therefore, the water movements reported in this article are outside the statistical uncertainty of the results.

\subsection{Application to lytic polysaccharide monooxygenase (LPMO)}

The primary use of quantum refinement is for systems for which the standard chemical restraints can be expected to be poor (for example metal sites or nonstandard ligands or inhibitors) or where standard refinement gives ambiguous results. To illustrate such a typical application, we have also tested the method on the neutron structure of a coppercontaining metalloenzyme, LPMO. In a recent study, Bacik and coworkers suggested that in subunit $B$ of the enzyme the terminal $\mathrm{N}$ atom, which coordinates the $\mathrm{Cu}$ atom, is partially deprotonated (Bacik et al., 2017). This was supported by the presence of an asymmetric nuclear difference-density peak around the $\mathrm{N}$-terminal atom. However, the interpretation is rather ambiguous, as the model was only refined against neutron data at $2.1 \AA$ resolution and the N-terminal deuterons were not modelled explicitly in the structure. Therefore, in order to discern whether a protonated or deprotonated N-terminus fits the data better, we performed joint QM refinement against both $\mathrm{X}$-ray and neutron data. This is suitable as the space groups and unit-cell parameters of both the X-ray and neutron crystals are nearly identical.

To determine the optimum weights, we performed a similar linear search to that performed for the deposited galectin structure. We kept the relative weight factors with $w_{\mathrm{X}} / w_{\mathrm{N}}=1$ (which are the default in phenix.refine) and increased them stepwise from $w_{\mathrm{X}}=1$ to $w_{\mathrm{X}}=10$. We then compared the RSZD scores of the residues in the quantum system (including the $\mathrm{Cu}$ atom and the peroxide molecule). All calculations were performed on subunit $A$, as this showed no ambiguity in the protonation of the N-terminus. As can be seen in Table 4, different weights did not change the fit of the structure to the data, with all RSZD scores being almost identical for the various weights. This is not completely surprising, as the QM system is quite rigid and the data quality is worse than for the galectin structure. However, if the weights are further decreased $\left(w_{\mathrm{N}}=w_{\mathrm{X}}=0.1\right.$ or smaller $)$ the RSZD increases significantly, showing that the structure becomes too biased towards QM. 
Table 4

Maximum absolute RSZD of the residues in the QM system in the QM refinement of subunit $A$ of the LPMO structure obtained with different weights of the experimental data $\left(w_{\mathrm{N}}\right.$ and $\left.w_{\mathrm{X}}\right)$.

Note that $w_{\mathrm{X}} / w_{\mathrm{N}}=1$ in all refinements.

\begin{tabular}{llllllllllllll}
\hline$w_{\mathrm{N}}$ & 0.001 & 0.01 & 0.1 & 1 & 2 & 3 & 4 & 5 & 6 & 7 & 8 & 9 & 10 \\
\hline$w_{\mathrm{X}}$ & 0.001 & 0.01 & 0.1 & 1 & 2 & 3 & 4 & 5 & 6 & 7 & 8 & 9 & 10 \\
\hline Copper & 1.2 & 1.2 & 1.2 & 1.2 & 1.2 & 1.3 & 1.2 & 1.2 & 1.2 & 1.2 & 1.2 & 1.2 & 1.2 \\
Peroxide & 0.1 & 0.1 & 0.1 & 0.1 & 0.1 & 0.1 & 0.1 & 0.1 & 0.1 & 0.1 & 0.1 & 0.1 & 0.1 \\
His32 & 2.5 & 2.4 & 2.1 & 0.7 & 0.7 & 0.7 & 0.7 & 0.8 & 0.8 & 0.8 & 0.8 & 0.8 & 0.8 \\
His109 & 1.0 & 1.0 & 0.9 & 0.2 & 0.2 & 0.2 & 0.3 & 0.3 & 0.3 & 0.3 & 0.3 & 0.3 & 0.3 \\
Phe164 & 0.5 & 0.5 & 0.5 & 0.5 & 0.5 & 0.5 & 0.5 & 0.5 & 0.5 & 0.5 & 0.5 & 0.5 & 0.5 \\
Wat-301 & 0.9 & 0.9 & 0.9 & 0.9 & 0.9 & 0.9 & 0.9 & 0.9 & 0.9 & 0.9 & 0.9 & 0.9 & 0.9 \\
Sum & $\mathbf{6 . 2}$ & $\mathbf{6 . 1}$ & $\mathbf{5 . 7}$ & $\mathbf{3 . 6}$ & $\mathbf{3 . 6}$ & $\mathbf{3 . 7}$ & $\mathbf{3 . 7}$ & $\mathbf{3 . 8}$ & $\mathbf{3 . 8}$ & $\mathbf{3 . 8}$ & $\mathbf{3 . 8}$ & $\mathbf{3 . 8}$ & $\mathbf{3 . 8}$
\end{tabular}

Table 5

Structure quality of the LPMO structure before and after QM refinement with the active site in subunit $B$ with two deuterons on the $\mathrm{N}$-terminus as the QM system.

$R_{\text {work }}$ and $R_{\text {free }}$ were calculated in $n C N S$. Geometry statistics were calculated with MolProbity.

\begin{tabular}{|c|c|c|}
\hline & Before & After \\
\hline$R_{\text {work }}(\mathrm{X}$-ray $)$ & 0.161 & 0.150 \\
\hline$R_{\text {free }}(\mathrm{X}$-ray $)$ & 0.164 & 0.153 \\
\hline$R_{\text {work }}$ (neutron) & 0.243 & 0.237 \\
\hline$R_{\text {free }}$ (neutron) & 0.248 & 0.244 \\
\hline R.m.s.d., bonds (®̊) & 0.009 & 0.008 \\
\hline R.m.s.d., angles $\left({ }^{\circ}\right)$ & 0.9 & 0.9 \\
\hline Ramachandran favoured (\%) & 98.5 & 99.2 \\
\hline Ramachandran allowed (\%) & 1.5 & 0.8 \\
\hline Ramachandran outliers (\%) & 0.0 & 0.0 \\
\hline Rotamer outliers (\%) & 0.0 & 0.0 \\
\hline All-atom clashscore & 1.82 & 2.93 \\
\hline
\end{tabular}

Therefore, we selected $w_{\mathrm{N}}=w_{\mathrm{X}}=1$ (which gave the lowest RSZD scores and will not bias the structure towards either

the QM or the crystallographic data) and performed a QM refinement with subunit $B$ in the quantum system. We compared the results of using two different models, one with the $\mathrm{N}$-terminus protonated (i.e. modelled as $-\mathrm{ND}_{2}$ ) and the other deprotonated (i.e. modelled as $-\mathrm{ND}^{-}$). Fig. 11 clearly shows a positive difference density close to the N-terminus in the deprotonated model (green grid in the centre of Fig. 11b), indicating that modelling the $\mathrm{N}$-terminus as a protonated $-\mathrm{ND}_{2}$ group is a better interpretation of the joint neutron and X-ray data. A more in-depth discussion of these calculations and their implication for the biological function of the enzyme has been published elsewhere (Caldararu et al., 2018).

The refinement statistics for the final QM-refined structure are shown in Table 5. As for the galectin structure, the overall structure of the protein keeps its integrity and the $R / R_{\text {free }}$ values are slightly improved compared with those calculated with $n C N S$ before the QM refinement.

\section{Conclusions}

In this study, we have implemented the combination of joint neutron and X-ray crystallographic refinement with QM/MM calculations. We have combined the freely available $n C N S$ software (Adams et al., 2009) with the QM software TURBOMOLE (Furche et al., 2014). We have applied the method to a recent neutron structure of lactose bound to the carbohydrate-recognition domain of galectin-3 (Manzoni et al., 2018), and to the active site of LPMO (Caldararu et al., 2018). We show that the method behaves properly and that we can use the two weight factors $w_{\mathrm{N}}$ and $w_{\mathrm{X}}$ to bias the structure towards the neutron data, the X-ray data or the QM structure. We can obtain an ideal compromise between the QM and the crystallographic methods by monitoring the RSZD values of residues in the QM system (see Tables 3 and 4), whereas optimizing the crystallographic

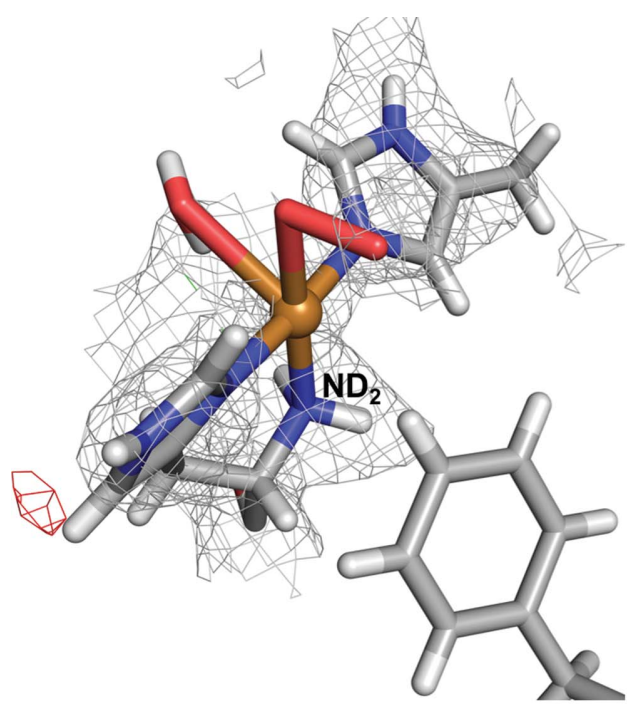

(a)

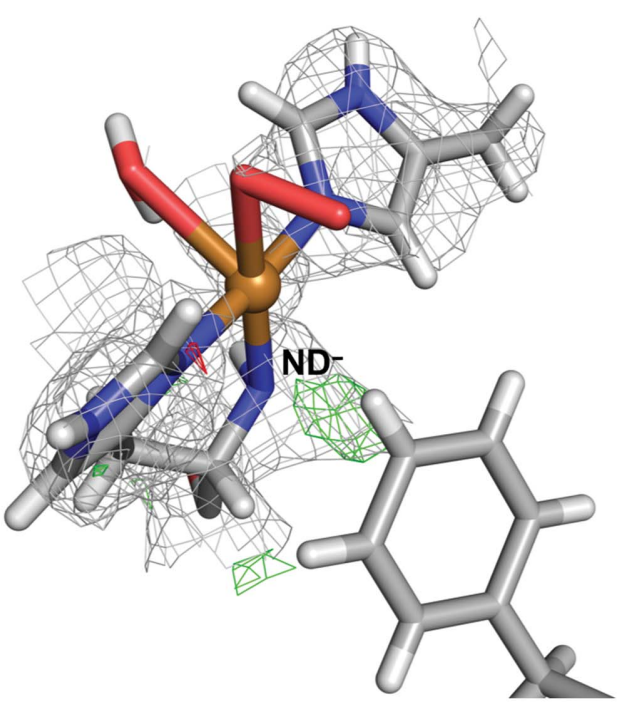

(b)

Figure 11

Structure and nuclear density maps of the active site of LPMO in subunit $B$ after QM refinement. $(a)$ The $\mathrm{N}$-terminus in the protonated $-\mathrm{ND}_{2}$ form and $(b)$ the $\mathrm{N}$-terminus in the deprotonated $\mathrm{ND}^{-}$form. The $2 m\left|F_{\mathrm{o}}\right|-D\left|F_{\mathrm{c}}\right|$ nuclear density at $1.0 \sigma$ is shown as a blue grid and $m\left|F_{\mathrm{o}}\right|-D\left|F_{\mathrm{c}}\right|$ nuclear difference density is shown at $3.0 \sigma$ (green grid) and $-3.0 \sigma$ (red grid).
$R_{\text {free }}$ values seems to give less reliable results.

The approach allows us to identify and remove problems in the corresponding structure optimized with conventional methods without the use of QM. In particular, the description of the hydrogen-bonding pattern is improved, with a level of detail and confidence that is not possible with standard refinement. For the solvent-accessible binding site in galectin-3, it is primarily the solvent molecules and the $\mathrm{D}$ atoms of the ligand that are improved by QM refinement. In several cases, the hydrogen-bonding pattern was modified to avoid steric clashes or to improve the hydrogen-bond strengths. For LPMO, we show 
that the method can be used to determine the protonation states of interesting residues by comparing the structures refined in the different states. Of course, the improvements are most important when the experimental data are poorer, as the QM refinements of the preliminary structures of galectin-3 and of LPMO showed.

There is no significant difference in either the $R$ values or the density maps between the normal and QM-refined structures, which means that both models essentially fit the crystallographic data equally well. However, the QM-refined models include more sophisticated chemical information and hence allow more confidence in drawing functional or mechanistic conclusions from the structure.

Naturally, this improvement comes with an additional cost. The QM refinements took around $5 \mathrm{~h}$ each, whereas standard neutron/X-ray refinement takes only a few minutes (both on a single processor). On the other hand, the $\mathrm{QM}$ system was quite large for the solvent-exposed site of galectin-3 (153 atoms). However, the method is intended to be used primarily at the end of the refinement procedure and only for sites of particular interest, as shown in the application to LPMO. Moreover, some hours of calculation are not much compared with the time required to grow the large crystals that are needed for neutron crystallography or even with the datacollection time.

A limitation of the present QM refinement approach is that it can only model a single conformation of the atoms in the QM system. This means that if a D atom or a water molecule may form several different hydrogen bonds of similar stability, the QM refinement will probably converge to one of these, typically that favoured by enthalpic factors and by the QM hydrogen-bond energy, unless the density maps show that the $\mathrm{D}$ atom is clearly in between. Naturally, this problem can be solved by simply using two separate QM calculations, one for each conformation, but this would double the computational cost and give an exponential increase in the number of QM systems if there is more than one alternative conformation.

A related problem is that the QM-refined structures will be biased towards the formation of hydrogen bonds within the QM system. The reason for this is that there are no electrostatic interactions with the MM surroundings in the current implementation (following the convention of neutron and $\mathrm{X}$-ray crystallography). The user needs to remember this problem when interpreting the structures. Technically, it is trivial to turn on the electrostatics in the calculations. However, this would introduce several conceptual problems. Firstly, the calculations will no longer be directly comparable with standard refinement, making the comparison more complicated. Secondly, all D atoms are not unambiguously discernible in the neutron structure. However, the electrostatics strongly depend on the position of these atoms, at least the polar D atoms. This makes it necessary to guess the positions of all $\mathrm{D}$ atoms. This can in principle be performed with standard MM software. However, it means that the final structure will be a mixture of observed and speculated atomic positions. Thirdly, alternative conformations will again be a problem, because the QM atoms can only interact with one set of charges. For these reasons, we have decided to not include electrostatics in this first version of the $\mathrm{QM}$ refinement procedure.

\section{Acknowledgements}

The computations were performed on computer resources provided by the Swedish National Infrastructure for Computing (SNIC) at LUNARC at Lund University.

\section{Funding information}

This investigation has been supported by grants from the Swedish Research Council (projects 2014-5540 and 201805003), from the Knut and Alice Wallenberg Foundation (KAW 2013.0022), the Royal Physiographic Society in Lund, from the European Spallation Source ERIC and from eSSENCE: the e-science collaboration.

\section{References}

Adams, P. D., Mustyakimov, M., Afonine, P. V. \& Langan, P. (2009). Acta Cryst. D65, 567-573.

Adams, P. D., Pannu, N. S., Read, R. J. \& Brünger, A. T. (1997). Proc. Natl Acad. Sci. USA, 94, 5018-5023.

Afonine, P. V., Grosse-Kunstleve, R. W., Echols, N., Headd, J. J., Moriarty, N. W., Mustyakimov, M., Terwilliger, T. C., Urzhumtsev, A., Zwart, P. H. \& Adams, P. D. (2012). Acta Cryst. D68, 352-367.

Afonine, P. V., Mustyakimov, M., Grosse-Kunstleve, R. W., Moriarty, N. W., Langan, P. \& Adams, P. D. (2010). Acta Cryst. D66, 11531163.

Antony, J., Sure, R. \& Grimme, S. (2015). Chem. Commun. 51, 1764 1774.

Bacik, J.-P., Mekasha, S., Forsberg, Z., Kovalevsky, A. Y., VaajeKolstad, G., Eijsink, V. G. H., Nix, J. C., Coates, L., Cuneo, M. J., Unkefer, J. \& Chen, J. C.-H. (2017). Biochemistry, 56, 2529-2532.

Blakeley, M. P. (2009). Crystallogr. Rev. 15, 157-218.

Blakeley, M. P., Teixeira, S. C. M., Petit-Haertlein, I., Hazemann, I., Mitschler, A., Haertlein, M., Howard, E. \& Podjarny, A. D. (2010). Acta Cryst. D66, 1198-1205.

Borbulevych, O., Martin, R. I., Tickle, I. J. \& Westerhoff, L. M. (2016). Acta Cryst. D72, 586-598.

Borbulevych, O. Y., Plumley, J. A., Martin, R. I., Merz, K. M. \& Westerhoff, L. M. (2014). Acta Cryst. D70, 1233-1247.

Brooks, B. R., Brooks, C. L., MacKerell, A. D., Nilsson, L., Petrella, R. J., Roux, B., Won, Y., Archontis, G., Bartels, C., Boresch, S., Caflisch, A., Caves, L., Cui, Q., Dinner, A. R., Feig, M., Fischer, S., Gao, J., Hodoscek, M., Im, W., Kuczera, K., Lazaridis, T., Ma, J., Ovchinnikov, V., Paci, E., Pastor, R. W., Post, C. B., Pu, J. Z., Schaefer, M., Tidor, B., Venable, R. M., Woodcock, H. L., Wu, X., Yang, W., York, D. M. \& Karplus, M. (2009). J. Comput. Chem. 30, 1545-1614.

Brünger, A. T., Adams, P. D., Clore, G. M., DeLano, W. L., Gros, P., Grosse-Kunstleve, R. W., Jiang, J.-S., Kuszewski, J., Nilges, M., Pannu, N. S., Read, R. J., Rice, L. M., Simonson, T. \& Warren, G. L. (1998). Acta Cryst. D54, 905-921.

Brünger, A. T., Karplus, M. \& Petsko, G. A. (1989). Acta Cryst. A45, 50-61.

Brünger, A. T. \& Rice, L. M. (1997). Methods Enzymol. 277, 243-269.

Caldararu, O., Oksanen, E., Ryde, U. \& Hedegård, E. D. (2018). Chem. Sci. 10, 576-586.

Cao, L. \& Ryde, U. (2018). Front. Chem. 6, 89.

Coppens, P. (1967). Science, 158, 1577-1579.

Cruickshank, D. W. J. (1999). Acta Cryst. D55, 583-601.

Delacour, D., Koch, A. \& Jacob, R. (2009). Traffic, 10, 1405-1413.

Engh, R. A. \& Huber, R. (1991). Acta Cryst. A47, 392-400. 
Fisher, S. Z., Anderson, S., Henning, R., Moffat, K., Langan, P., Thiyagarajan, P. \& Schultz, A. J. (2007). Acta Cryst. D63, 11781184.

Furche, F., Ahlrichs, R., Hättig, C., Klopper, W., Sierka, M. \& Weigend, F. (2014). WIREs Comput. Mol. Sci. 4, 91-100.

Genoni, A., Bučinský, L., Claiser, N., Contreras-García, J., Dittrich, B., Dominiak, P. M., Espinosa, E., Gatti, C., Giannozzi, P., Gillet, J.-M., Jayatilaka, D., Macchi, P., Madsen, A. Ø., Massa, L., Matta, C. F., Merz, K. M. Jr, Nakashima, P. N. H., Ott, H., Ryde, U., Schwarz, K., Sierka, M. \& Grabowsky, S. (2018). Chem. Eur. J. 24, 10881-10905.

Grigorian, A. \& Demetriou, M. (2010). Methods Enzymol. 480, 245266.

Grimme, S., Antony, J., Ehrlich, S. \& Krieg, H. (2010). J. Chem. Phys. 132, 154104.

Harris, P. V., Xu, F., Kreel, N. E., Kang, C. \& Fukuyama, S. (2014). Curr. Opin. Chem. Biol. 19, 162-170.

Hsiao, Y., Sanchez-Garcia, E., Doerr, M. \& Thiel, W. (2010). J. Phys. Chem. B, 114, 15413-15423.

Johannes, L., Jacob, R. \& Leffler, H. (2018). J. Cell Sci. 131, jcs208884.

Leffler, H., Carlsson, S., Hedlund, M., Qian, Y. \& Poirier, F. (2002). Glycoconj. J. 19, 433-440.

Liu, F.-T. \& Rabinovich, G. A. (2010). Ann. N. Y. Acad. Sci. 1183, 158-182.

MacKinnon, A. C., Farnworth, S. L., Hodkinson, P. S., Henderson, N. C., Atkinson, K. M., Leffler, H., Nilsson, U. J., Haslett, C., Forbes, S. J. \& Sethi, T. (2008). J. Immunol. 180, 2650-2658.

Manzoni, F., Saraboji, K., Sprenger, J., Kumar, R., Noresson, A.-L., Nilsson, U. J., Leffler, H., Fisher, S. Z., Schrader, T. E., Ostermann, A., Coates, L., Blakeley, M. P., Oksanen, E. \& Logan, D. T. (2016). Acta Cryst. D72, 1194-1202.

Manzoni, F., Wallerstein, J., Schrader, T. E., Ostermann, A., Coates, L., Akke, M., Blakeley, M. P., Oksanen, E. \& Logan, D. T. (2018). J. Med. Chem. 61, 4412-4420.

Murshudov, G. N., Skubák, P., Lebedev, A. A., Pannu, N. S., Steiner, R. A., Nicholls, R. A., Winn, M. D., Long, F. \& Vagin, A. A. (2011). Acta Cryst. D67, 355-367.

Neese, F. (2006). J. Biol. Inorg. Chem. 11, 702-711.

Nilsson, K., Lecerof, D., Sigfridsson, E. \& Ryde, U. (2003). Acta Cryst. D59, 274-289.

Nilsson, K. \& Ryde, U. (2004). J. Inorg. Biochem. 98, 1539-1546.

Orpen, A. G., Pippard, D., Sheldrick, G. M. \& Rouse, K. D. (1978). Acta Cryst. B34, 2466-2472.

Pannu, N. S. \& Read, R. J. (1996). Acta Cryst. A52, 659-668.

Quinlan, R. J., Sweeney, M. D., Lo Leggio, L., Otten, H., Poulsen, J. N., Johansen, K. S., Krogh, K. B. R. M., Jørgensen, C. I., Tovborg, M., Anthonsen, A., Tryfona, T., Walter, C. P., Dupree, P., Xu, F.,
Davies, G. J. \& Walton, P. H. (2011). Proc. Natl Acad. Sci. USA, 108, 15079-15084.

Rabinovich, G. A., Liu, F.-T., Hirashima, M. \& Anderson, A. (2007). Scand. J. Immunol. 66, 143-158.

Reynolds, C. H. (2014). ACS Med. Chem. Lett. 5, 727-729.

Rulíšek, L. \& Ryde, U. (2006). J. Phys. Chem. B, 110, 1151111518.

Ryde, U. (1996). J. Comput. Aided Mol. Des. 10, 153-164.

Ryde, U. (2007). Dalton Trans., pp. 607-625.

Ryde, U. (2016). Methods Enzymol. 577, 119-158.

Ryde, U. \& Nilsson, K. (2003). J. Am. Chem. Soc. 125, 1423214233.

Ryde, U., Olsen, L. \& Nilsson, K. (2002). J. Comput. Chem. 23, 10581070.

Ryde, U. \& Olsson, M. H. M. (2001). Int. J. Quantum Chem. 81, 335347.

Saraboji, K., Håkansson, M., Genheden, S., Diehl, C., Qvist, J., Weininger, U., Nilsson, U. J., Leffler, H., Ryde, U., Akke, M. \& Logan, D. T. (2012). Biochemistry, 51, 296-306.

Schäfer, A., Horn, H. \& Ahlrichs, R. (1992). J. Chem. Phys. 97, 25712577.

Sears, V. F. (1986). Methods Exp. Phys. 23A, 521-550.

Söderhjelm, P. \& Ryde, U. (2006). J. Mol. Struct. Theochem, 770, 199219.

Sure, R. \& Grimme, S. (2015). J. Chem. Theory Comput. 11, 37853801.

Tao, J., Perdew, J. P., Staroverov, V. N. \& Scuseria, G. E. (2003). Phys. Rev. Lett. 91, 146401.

Tickle, I. J. (2012). Acta Cryst. D68, 454-467.

Vaaje-Kolstad, G., Westereng, B., Horn, S. J., Liu, Z., Zhai, H., Sørlie, M. \& Eijsink, V. G. H. (2010). Science, 330, 219-222.

Winn, M. D., Ballard, C. C., Cowtan, K. D., Dodson, E. J., Emsley, P., Evans, P. R., Keegan, R. M., Krissinel, E. B., Leslie, A. G. W., McCoy, A., McNicholas, S. J., Murshudov, G. N., Pannu, N. S., Potterton, E. A., Powell, H. R., Read, R. J., Vagin, A. \& Wilson, K. S. (2011). Acta Cryst. D67, 235-242.

Wlodawer, A. (1980). Acta Cryst. B36, 1826-1831.

Wlodawer, A. \& Hendrickson, W. A. (1982). Acta Cryst. A38, 239247.

Wlodawer, A. \& Sjölin, L. (1982). Proc. Natl Acad. Sci. USA, 79, 1418-1422.

Yu, N., Yennawar, H. P. \& Merz, K. M. (2005). Acta Cryst. D61, 322 332.

Zheng, M., Moriarty, N. W., Xu, Y., Reimers, J. R., Afonine, P. V. \& Waller, M. P. (2017). Acta Cryst. D73, 1020-1028.

Zheng, M., Reimers, J. R., Waller, M. P. \& Afonine, P. V. (2017). Acta Cryst. D73, 45-52. 